TSUKUBA J. MATH.

Vol. 15 No. 2 (1991), $351-387$

\title{
A FACTOR OF SINGULAR HOMOLOGY
}

\author{
By \\ Katsuya EDA and Katsuro SAKAI
}

\section{Introduction}

Singular homology is a beautiful theory, in which we can see a clear correspondence between Algebra and Topology. However, it behaves badly on topological spaces which are not locally simply connected in comparison with Čech homology. In the present paper we introduce a canonical factor $H_{n}^{T}(X)$ of singular homology $H_{n}(X)$, which agrees with singular homology on ANR's and behaves well on the indicated spaces. We also introduce a notion "quasihomotopy" for continuous maps. It turns out that the factor is invariant under quasi-homotopy.

We state definitions and basic facts in Section 1. In Section 2, we prove that $H_{n}^{T}(X)=H_{n}(X)$ for every ANR $X$. In Section 3, we show that $H_{0}^{T}(X)$ is isomorphic to a free abelian group whose rank is equal to the cardinality of equivalence classes with respect to a certain kind of connectedness. There we also introduce a notion "quasi-homotopy" and show that the factor is invariant under quasi-homotopy. In Sections 4, 5 and 6, one can see the advantage of $H_{n}^{T}(X)$ to the singular homology groups $H_{n}(X)$. More precisely, $H_{n}^{T}(X)$ are calculated for certain spaces such as the so-called Hawaiian earring and infinite products and so on. Furthermore, certain natural abelian groups are realized as $H_{n}^{T}(X)$ by natural topological spaces $X$. We shall show that any homomorphism from $H_{n}^{T}(X)$ to $H_{n}^{T}(Y)$ is induced by a continuous map if $X$ and $Y$ are obtained by attaching copies of $\boldsymbol{S}^{n}$ in certain ways. For example, for the Hawaiian earring $\boldsymbol{H}$, any homomorphism from $H_{1}^{T}(\boldsymbol{H})$ to itself can be induced by a continuous map from $\boldsymbol{H}$ to itself, though this fact does not hold for $H_{1}(\boldsymbol{H})$. We also characterize slenderness of abelian groups by using $H_{1}^{T}(\boldsymbol{H})$ and the notion of spatial homomorphism. (See Theorems 6.2, 6.3 and Remark 6.4.) Ralph [22] has defined a factor of singular chain and homology groups $H A$ and $H M$. In Section 4 , one can see that $H_{n}^{T}$ has similar effect as $H A$.

Received July 14, 1989. Revised January 21, 1991. 


\section{Definitions and basic facts}

All (topological) spaces in this paper are Tychonoff spaces and all groups are abelian, unless otherwise stated. For a space $X$, let $\operatorname{Pm}(X)$ denote the set of all continuous pseudo-metrics on $X$. ANR's mean absolute neighborhood retracts for metrizable spaces [13].

By $\Delta_{n}$, we denote the standard $n$-simplex with vertices $\boldsymbol{e}_{0}, \cdots, \boldsymbol{e}_{n}$, where $\boldsymbol{e}_{i}(i)=1$ and $\boldsymbol{e}_{i}(j)=0$ for $j \neq i$. We regard $\Delta_{n} \subset \Delta_{n+1}$. Let $C\left(\Delta_{n}, X\right)$ be the set of all continuous maps from the $n$-simplex $\Delta_{n}$ to a topological space $X, S_{n}(X)$ the free abelian group generated by $C\left(\Delta_{n}, X\right)$ and $\partial=\partial_{n+1}: S_{n+1}(X) \rightarrow S_{n}(X)$ be the boundary operator as usual [3, Ch. III 2.1]. Namely $\varepsilon_{i}: \Delta_{n} \rightarrow \Delta_{n+1}$ is the linear map defined by : $\varepsilon_{i}\left(\boldsymbol{e}_{j}\right)=\boldsymbol{e}_{j}$ for $j<i$ and $\varepsilon_{i}\left(\boldsymbol{e}_{j}\right)=\boldsymbol{e}_{j+1}$ for $j \geqq i$. Then $\partial_{n+1}(u)$ $=\sum_{i=0}^{n+1}(-1)^{i} u \circ \varepsilon_{i}$ for $u \in C\left(\Delta_{n+1}, X\right)$. The singular homology is defined by: $H_{n}(X)=Z_{n}(X) / B_{n}(X)$, where $Z_{n}(X)=\operatorname{Ker} \partial_{n}$ and $B_{n}(X)=\operatorname{Im} \partial_{n+1}$.

Now we state the definition of the factor. Regarding $C\left(\Delta_{n}, X\right)$ as the topological space with the compact-open topology, we consider $S_{n}(X)$ as the free abelian topological group on $C\left(\Delta_{n}, X\right)$ (in the sence of Markov [18]). Then, $\partial$ becomes a continuous homomorphism, hence the closure $\overline{B_{n}(X)}$ of $B_{n}(X)$ is included in $Z_{n}(X)$. (Refer to [18], [12] and [17] for the definition of free abelian topological groups and [20] for summary.) We define $H_{n}^{T}(X)=Z_{n}(X) / \overline{B_{n}(X)}$. Though $H_{n}^{T}(X)$ naturally becomes a topological group, we ignore this topology. By definition, there exists a natural epimorphism $\sigma_{X}: H_{n}(X) \rightarrow H_{n}^{T}(X)$. Let $f_{*}: H_{n}(X) \rightarrow H_{n}(Y)$ be the homomorphism induced by a continuous map $f: X \rightarrow Y$.

Proposition 1.1. Each continuous map $f: X \rightarrow Y$ induces a homomorphism $f_{*}^{T}: H_{n}^{T}(X) \rightarrow H_{n}^{T}(Y)$ such that $f_{*}^{T} \circ \sigma_{X}=\sigma_{Y} \circ f_{*}$.

In fact, since $S_{n}(X)$ is a free abelian topological group on $C\left(\Delta_{n}, X\right)$, we can define a continuous homomorphism $f_{\#}: S_{n}(X) \rightarrow S_{n}(Y)$ by: $f_{\#}(u)=f \circ u \in$ $C\left(\Delta_{n}, X\right)$ for $u \in C\left(\Delta_{n}, X\right)$. Then $f_{\#}\left(\overline{\left.B_{n}(X)\right)} \subset \overline{f_{\#}\left(B_{n}(X)\right.}\right) \subset \overline{B_{n}(Y)}$ by the continuity of $f_{\#}$ and a basic fact about singular homology. Thus $f_{*}^{T}$ can be defined by $f_{*}^{T}\left(u+\overline{B_{n}(X)}\right)=f_{\#}(u)+\overline{B_{n}(Y)}$. We have $f_{*}^{T} \sigma_{X}=\sigma_{Y^{\circ}} f_{*}$ by definition.

By the homotopy invariance of singular homology and Proposition 1.1 and by definition, we get the following.

PROPOSITION 1.2. The groups $H_{n}^{T}(X)$ are homotopy invariant.

Proposition 1.3. If two continuous maps $f, g: X \rightarrow Y$ are homotopic, then 
$f_{*}^{T}=g_{*}^{T}$.

This will be strengthened in Section 3 (Theorem 3.8). For basic results and notions about algebraic topology, we refer the reader to [3 and 23]. The set of nonzero positive integers is denoted by $N$. As usual we abbreviate the subscript of the boundary operator $\partial_{n}$ and $Z_{n}(X)$ or $B_{n}(X)$ by $Z_{n}$ or $B_{n}$ respectively, in case no confusion will occur.

\section{2. $H_{n}^{T}(X)=H_{n}(X)$ for ANR's $X$}

The purpose of this section is to prove the following theorem.

THEOREM 2.1. Let $X$ be an $A N R$. Then $B_{n}(X)$ is closed in the free abelian topological group $S_{n}(X)$ for each $n$. Consequently, $H_{n}^{T}(X)$ is identical with $H_{n}(X)$ for each $n$.

COROLlay 2.2. Let $X$ be a space which has the homotopy type of an ANR. Then $H_{n}^{T}(X)=H_{n}(X)$.

To show this, Graev's metric on free abelian groups plays a crucial role in spite of the fact that the metric topology is coarser than the free topology except rare cases. It seems impossible to perform the proof only by using the universality of free abelian topological groups [17]. Our proof implies that $B_{n}(X)$ is not only closed but also open in $Z_{n}(X)$ and hence $H_{n}^{T}(X)$ turns out to be discrete for every ANR $X$ even if we consider its topology.

First we introduce Graev's metric of the free abelian group $A(M)$ generated by a metric space $M=(M, \rho)[12]$. Fix an element $x^{*}$ of $M$ and extend $\rho$ to $\rho^{\prime}$ on the set $M \cup\{0\} \cup-M$ as follows: (1) $\rho^{\prime}\left(x^{*}, 0\right)=1 ;(2) \rho^{\prime}(x, 0)=\rho^{\prime}(-x, 0)$ $=\rho\left(x, x^{*}\right)+1$ for $x \in X$; (3) $\rho^{\prime}(-x,-y)=\rho(x, y)$ and $\rho^{\prime}(-x, y)=\rho(x, 0)+\rho(0, y)$ for $x, y \in M$. For $u, v \in A(M)$, define

$$
\begin{aligned}
\tilde{\rho}(u, v)=\inf \left\{\sum_{i=0}^{m} \rho^{\prime}\left(x_{i}, y_{i}\right): u=\sum_{i=0}^{m} x_{i}, v=\sum_{i=0}^{m} y_{i},\right. \\
\left.x_{i}, y_{i} \in M \cup\{0\} \cup-M, m \in N\right\} .
\end{aligned}
$$

In fact, Graev [12] proved that there exist $x_{i}, y_{i} \in M \cup\{0\} \cup-M(0 \leqq i \leqq m)$ such that $u=\sum_{i=0}^{m} x_{i}, v=\sum_{i=y_{i}}^{m}$ and $\tilde{\rho}(u, v)=\sum_{i=0}^{m} \rho^{\prime}\left(x_{i}, y_{i}\right)$ and that $\tilde{\rho}$ is a metric on $A(M)$. Observe that both $x_{i}$ and $y_{i}$ in the above belong to either $M$ or $-M$, if $\tilde{\rho}(u, v)<1$. One should remark that the topology induced by $\tilde{\rho}$ is coarser than the topology of $A(M)$ as the free abelian topological group on $M$. For the simplicity of notation, we also use $\rho$ for $\tilde{\rho}$. 
Now we start to prove the theorem. Without loss of generality, we can assume by [19] that $X$ is a closed subset of a normed linear space $Y=(Y,\|\cdot\|)$ and there is a retraction $r: U \rightarrow X$ of a uniform neighborhood $U$ of $X$ in $Y$, i.e.,

$$
U=\{y \in Y:\|x-y\|<\delta \text { for some } x \in X\}
$$

for some $\delta>0$. The compact-open topology on $C\left(\Delta_{n}, X\right)$ is induced by the supmetric

$$
\rho(f, g)=\sup \left\{\|f(\alpha)-g(\alpha)\|: \alpha \in \Delta_{n}\right\}
$$

Let $\rho(=\tilde{\rho})$ be the metric on $S_{n}(X)$ induced by $\rho$ as above. To prove the theorem, it suffices to show that $B_{n}(X)$ is open in $Z_{n}(X)$, hence closed under this metric $\rho$.

To this end, let $u \in Z_{n}(X)$ and $v \in B_{n}(X)$ such that $\rho(u, v)<\min \{1, \delta / N\}$, where $N=\max \left\{\left(\begin{array}{c}n+1 \\ j\end{array}\right): 0 \leqq j \leqq n+1\right\}$. By the remark following the definition of $\rho(=\tilde{\rho})$, we can write $u=\sum_{k=0}^{m} \lambda_{k} u_{k}$ and $v=\sum_{k=0}^{m} \lambda_{k} v_{k}$ where $u_{k}, v_{k} \in C\left(\Delta_{n}, X\right)$, $\lambda_{k}= \pm 1$ and $\rho(u, v)=\sum_{k=0}^{m} \rho\left(u_{k}, v_{k}\right)<\delta / N$. We will construct $\sigma \in S_{n+1}(U)$ so that $\partial \sigma=u-v$. Then $r_{\#}(\sigma) \in S_{n+1}(X)$ and $\partial\left(r_{\#}(\sigma)\right)=r_{\#}(\partial \sigma)=r_{\#}(u-v)=u-v$ because $u-v \in S_{n}(X)$. Thus it follows $u=\partial\left(r_{\#}(\sigma)\right)+v \in B_{n}(X)$.

Since $\partial u=\sum_{k=0}^{m} \sum_{i=0}^{n}(-1)^{i} \lambda_{k} u_{k} \varepsilon_{i}=0$, we have a partition $P_{u}$ of the set $(m+1)$ $\times(n+1)$ such that each member of $P_{u}$ has exactly two elements and $\left\{(k, i),\left(k^{\prime}, i^{\prime}\right)\right\} \in P_{u}$ implies $(-1)^{i} \lambda_{k} u_{k} \varepsilon_{i}+(-1)^{i^{\prime}} \lambda_{k^{\prime}} u_{k^{\prime}} \varepsilon_{i^{\prime}}=0$, i. e., $(-1)^{i} \lambda_{k}+(-1)^{i^{\prime}} \lambda_{k^{\prime}}$ $=0$ and $u_{k} \varepsilon_{i}=u_{k^{\prime}} \varepsilon_{i^{\prime}}$. (We identify a natural number $n$ with the set $\{i: 0 \leqq i \leqq$ $n-1\}$ to simplify the notation.) We denote $(k, i) \sim_{u}\left(k^{\prime}, i^{\prime}\right)$ if $\left\{(k, i),\left(k^{\prime}, i^{\prime}\right)\right\}$ $\in P_{u}$. Similarly, we have a relation $\sim_{v}$ on $(m+1) \times(n-1)$ such that $(k, i) \sim_{v}$ $\left(k^{\prime}, i^{\prime}\right)$ implies $(-1)^{i} \lambda_{k} v_{k} \varepsilon_{i}+(-1)^{i^{\prime}} \lambda_{k^{\prime}} v_{k^{\prime}} \varepsilon_{i^{\prime}}=0$. Next, we extends $\sim_{u}$ and $\sim_{v}$ to relations on $(m+1) \times\{F: F \subset n+1$ and $|F|=j\}$ for $0 \leqq j \leqq n$ as follows: $(k, F) \sim_{u}$ $\left(k^{\prime}, F^{\prime}\right)$ if there exist $i \in F$ and $i^{\prime} \in F^{\prime}$ such that $(k, i) \sim{ }_{u}\left(k^{\prime}, i^{\prime}\right)$ and $g(F \backslash\{i\})=$ $F^{\prime} \backslash\left\{i^{\prime}\right\}$ for the order preserving (o.p.) bijection $g: n+1 \backslash\{i\} \rightarrow n+1 \backslash\left\{i^{\prime}\right\}$. For $F \subset n+1$, let $\varepsilon_{F}: \Delta_{n-|F|} \rightarrow \Delta_{n}$ be the linear map such that $\varepsilon_{F}\left(\boldsymbol{e}_{i}\right)=\boldsymbol{e}_{f(i)}$, where $f: n+1-|F| \rightarrow n+1 \backslash F$ is the o.p. bijection. Since $(k, i) \sim_{u}\left(k^{\prime}, i^{\prime}\right)$ implies $u_{k} \varepsilon_{i}$ $=u_{k^{\prime}} \varepsilon_{i^{\prime}},(k, F) \sim_{u}\left(k^{\prime}, F^{\prime}\right)$ implies $u_{k} \varepsilon_{F}=u_{k^{\prime}} \varepsilon_{F^{\prime}}$. Since for each $(k, i)$ there exists a unique $\left(k^{\prime}, i^{\prime}\right)$ such that $(k, i) \sim_{u}\left(k^{\prime}, i^{\prime}\right),\left|\left\{\left(k^{\prime}, F^{\prime}\right):\left(k^{\prime}, F^{\prime}\right) \sim_{u}(k, F)\right\}\right|$ $<|F|$. Extend $\sim_{v}$ similarly, which has the same properties as $\sim_{u}$. A $j$-block $B$ is a nonempty minimal subset of $(m+1) \times\{F: F \subset n+1$ and $|F|=j\}$ which satisfies the following: If $(k, F) \sim_{u}\left(k^{\prime}, F^{\prime}\right)$ or $(k, F) \sim_{v}\left(k^{\prime}, F^{\prime}\right)$ then $(k, F) \in B$ implies $\left(k^{\prime}, F^{\prime}\right) \in B$. In other words, $B$ is an equivalence class of the equivalence relation generated by $\sim_{u}$ and $\sim_{v}$. For the convenience of notation, 
we regard $\{(k, \varnothing)\}$ as a 0 -block for each $k$. For each block $B$, we choose a representative $(k(B), \mathscr{F}(B)) \in B$. Note that every $(k, F)$ belongs to only one $j$ block, where $j=|F|$.

Lemma 2.3. Suppose that $(k, F)$ and $\left(k^{\prime}, F^{\prime}\right)$ belong to the same $j$-block $B$, $F \subset G \subset n+1$ and $G^{\prime}=F^{\prime} \cup g(G \backslash F)$ for the o.p. injection $g: n+1 \backslash F \rightarrow n+1 \backslash F^{\prime}$. Then $(k, G)$ and $\left(k^{\prime}, G^{\prime}\right)$ belong to the same $|G|$-block.

Prooof. The case $j=0$ is clear. Let $j>0$. Note that $(k, F)$ and $\left(k^{\prime}, F^{\prime}\right)$ are combined by a sequence of members of $B$ such that adjoining members have the relations $\sim_{u}$ or $\sim_{v}$. We prove the lemma by induction on the length of this sequence. Suppose that the lemma holds for $(k, F)$ and $\left(k^{*}, F^{*}\right)$, i.e., $(k, G)$ and $\left(k^{*}, G^{*}\right)$ belong to the same $|G|$-block, where $G^{*}=F^{*} \cup g^{*}(G \backslash F)$ for the o.p. bijection $g^{*}: n+1 \backslash F \rightarrow n+1 \backslash F^{*}$. Now let $\left(k^{*}, F^{*}\right) \sim_{x}\left(k^{\prime}, F^{\prime}\right)$, where $x=u$ or $v$. By definition, there exist $i^{*} \in F^{*}$ and $i^{\prime} \in F^{\prime}$ such that $\left(k^{*}, i^{*}\right) \sim_{x}$ $\left(k^{\prime}, i^{\prime}\right)$ and $F^{\prime}=\left\{i^{\prime}\right\} \cup f\left(F^{*} \backslash\left\{i^{*}\right\}\right)$ for the o. p. bijection $f: n+1 \backslash\left\{i^{*}\right\} \rightarrow n+1 \backslash\left\{i^{\prime}\right\}$. Observe $g=f \circ g^{*}$. Then it follows

$$
\begin{aligned}
G^{\prime} & =F^{\prime} \cup f \circ g^{*}(G \backslash F) \\
& =\left\{i^{\prime}\right\} \cup f\left(F_{*} \backslash\left\{i^{*}\right\}\right) \cup f\left(G^{*} \backslash F^{*}\right) \\
& =\left\{i^{\prime}\right\} \cup f\left(G^{*} \backslash\left\{i^{*}\right\}\right) .
\end{aligned}
$$

Therefore $\left(k^{*}, G^{*}\right) \sim_{x}\left(k^{\prime}, G^{\prime}\right)$. Thus $(k, G)$ and $\left(k^{\prime}, G^{\prime}\right)$ belong to the same block.

LEMMA 2.4. If $(k, F)$ and $\left(k^{\prime}, F^{\prime}\right)$ belong to the same j-block $B$, then $\rho\left(u_{k} \varepsilon_{F}, u_{k^{\prime}} \varepsilon_{F^{\prime}}\right)<\delta, \rho\left(u_{k} \varepsilon_{F}, v_{k^{\prime}} \varepsilon_{F^{\prime}}\right)<\delta$ and $\rho\left(v_{k} \varepsilon_{F}, v_{k^{\prime}} \varepsilon_{F^{\prime}}\right)<\delta$.

Proof. Observe the following facts: For each $0 \leqq h \leqq m,|\{G:(h, G) \in B\}|$ $\leqq\left(\begin{array}{c}n+1 \\ j\end{array}\right) ;(h, G) \sim_{u}\left(h^{\prime}, G^{\prime}\right)$ implies $u_{h} \varepsilon_{G}=u_{h^{\prime}} \varepsilon_{G^{\prime}} ;(h, G) \sim_{v}\left(h^{\prime}, G^{\prime}\right)$ implies $v_{h} \varepsilon_{G}$ $=v_{h^{\prime}} \varepsilon_{G^{\prime}} ; \rho\left(u_{h} \varepsilon_{G}, v_{h} \varepsilon_{G}\right) \leqq \rho\left(u_{h}, v_{h}\right)$. Considering a shortest sequence combining $(k, F)$ and $\left(k^{\prime}, F^{\prime}\right)$ by $\sim_{u}$ and $\sim_{v}$, we obtain

$$
\begin{aligned}
\rho\left(u_{k} \varepsilon_{F}, u_{k^{\prime}} \varepsilon_{F^{\prime}}\right) & \leqq\left(\begin{array}{c}
n+1 \\
j
\end{array}\right) \sum_{h=0}^{m} \rho\left(u_{h}, v_{h}\right) \leqq\left(\begin{array}{c}
n+1 \\
j
\end{array}\right) \rho(u, v) \\
& <\left(\begin{array}{c}
n+1 \\
j
\end{array}\right) \delta / N \leqq \delta
\end{aligned}
$$

and the others similarly. 
Let $\varepsilon_{i}^{0}=\varepsilon_{i} \times \mathrm{id}: \Delta_{m-1} \times \Delta_{n} \rightarrow \Delta_{m} \times \Delta_{n}(0 \leqq i \leqq m)$ and $\varepsilon_{i}^{1}=\mathrm{id} \times \varepsilon_{i}: \Delta_{m} \times \Delta_{n-1} \rightarrow \Delta_{m} \times \Delta_{n}$ $(0 \leqq i \leqq n)$. For each $0 \leqq j \leqq n$, the set of all injections $s: j \rightarrow n+1$ is denoted by $I(j, n+1)$. Here, we admit $\varnothing$ as a map from 0 and identify $s$ with the $j$-tuple $\left(s_{0}, \cdots, s_{j-1}\right)$. For each $s \in I(j, n+1)$ and $0 \leqq p \leqq j$, let $f_{s}^{p}: n+1 \backslash\left\{s_{q}: 0 \leqq q<p\right\} \rightarrow$ $n+1-p$ be the o.p. bijection.

For each $k$, we define $\tau_{\varnothing}^{k}: \Delta_{n} \times \Delta_{1} \rightarrow Y$ by :

$$
\tau_{\varnothing}^{k}\left(\alpha,(1-\lambda) e_{0}+\lambda e_{1}\right)=(1-\lambda) \cdot v_{k}(\alpha)+\lambda \cdot u_{k}(\alpha)
$$

For each $s \in I(j, n+1)(j>0)$, we define inductively $\tau_{s}^{k}: \Delta_{n-j} \times \Delta_{j+1} \rightarrow Y$ as follows : $\tau_{s}^{k}(\alpha, \cdot)$ is linear for each $\alpha \in \Delta_{n-j}$ as a map from $\Delta_{j+1}$ to $Y$,

$$
\begin{aligned}
& \tau_{s}^{k}\left(\alpha, \boldsymbol{e}_{0}\right)=v_{k(B)}{ }^{\circ} \varepsilon_{\mathcal{F}(B)}(\alpha) \quad \text { and } \\
& \tau_{s}^{k}\left(\alpha, \boldsymbol{e}_{p+1}\right)=\tau_{\underline{s}}^{k}\left(\varepsilon_{f_{s}^{j-1}\left(s_{j-1}\right)}(\alpha), \boldsymbol{e}_{p}\right) \quad \text { for } 0 \leqq p \leqq j,
\end{aligned}
$$

where $\underline{s}=\left(s_{0}, \cdots, s_{j-2}\right)$ and $B$ is the $j$-block which contains $(k, \operatorname{Im} s)$.

We represent $\tau_{s}^{k}$ a little bit more directly by using the notion of blocks. Let $B_{s}^{k, p}$ be the $(j-p)$-block which contains $\left(k,\left\{s_{q}: 0 \leqq q<j-p\right\}\right)$ and $F_{s}^{k, p}=$ $\mathscr{F}\left(B_{s}^{k, p}\right) \cup\left\{g\left(s_{q}\right): j-p \leqq q<j\right\}$, where $g: n+1 \backslash\left\{s_{q}: 0 \leqq q<j-p\right\} \rightarrow n+1 \backslash \mathscr{F}\left(B_{s}^{k, p}\right)$ is the o.p. bijection. Then, $B_{s}^{k, 0}=B$ and $B_{s}^{k, p+1}=B_{\underline{s}}^{k, p}$ for $p \geqq 0$. Moreover, $F_{s}^{k, p+1}$ $=F_{\underline{s}}^{k, p} \cup\left\{g\left(s_{j-1}\right)\right\}$. Then by induction, we have the following.

LEMMA 2.5. For each $s \in I(j, n+1)$ and $0 \leqq p \leqq j \leqq n$,

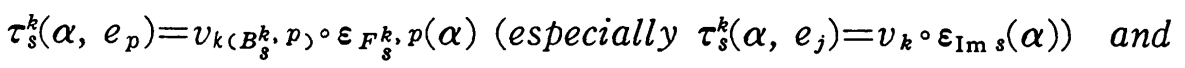

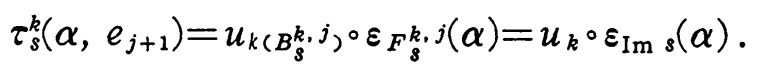

By Lemmas 2.3, 2.4 and 2.5 we have

CORLLARY 2.6. For each $s \in I(j, n+1)(0 \leqq j \leqq n), \operatorname{Im} \tau_{s}^{k} \subset U$.

Next we explicitly represent triangulations of products of simplexes. The order $\leqq$ on $(m+1) \times(n+1)$ is defined by : $(i, j) \leqq\left(i^{\prime}, j^{\prime}\right)$ if $i \leqq i^{\prime}$ and $j \leqq j^{\prime}$. We triangulate $\Delta_{m} \times \Delta_{n}$ so that vertices are $\left(\boldsymbol{e}_{i}, \boldsymbol{e}_{j}\right)$ and simplexes are spanned by $\left(\boldsymbol{e}_{i_{0}}, \boldsymbol{e}_{j_{0}}\right), \cdots,\left(\boldsymbol{e}_{i_{p}}, \boldsymbol{e}_{j_{p}}\right)$ with $\left(i_{0}, j_{0}\right)<\cdots<\left(i_{p}, j_{p}\right)$. By $O(m, n)$ we denote the set of all o.p. injections from $m+n+1$ to $(m+1) \times(n+1)$. For $t \in O(m, n)$, let $\mu_{t}: \Delta_{m+n} \rightarrow \Delta_{m} \times \Delta_{n}$ be the linear map defined by $\mu_{t}\left(\boldsymbol{e}_{i}\right)=\left(\boldsymbol{e}_{t_{0}(i)}, \boldsymbol{e}_{t_{1}(i)}\right)$, where $t(i)$ $=\left(t_{0}(i), t_{1}(i)\right)$.

For $\tau: \Delta_{m} \times \Delta_{n} \rightarrow U(m, n \geqq 0)$, let 


$$
C_{m, n}(\tau)=\sum_{t \in O(m, n)}(-1)^{c(t)} \tau \circ \mu_{t},
$$

where $c(t)=\sum_{j=0}^{m+n-1} t_{0}(j)\left(t_{1}(j+1)-t_{1}(j)\right)$,

and especially $C_{-1, n}(\tau)=C_{m,-1}(\tau)=0$. The following figure helps us to prove the next lemma. In the figure, $t \in O(m, n)$ is written as a shortest path from $(0,0)$ to $(m, n)$ on the lattice.

(Figure)

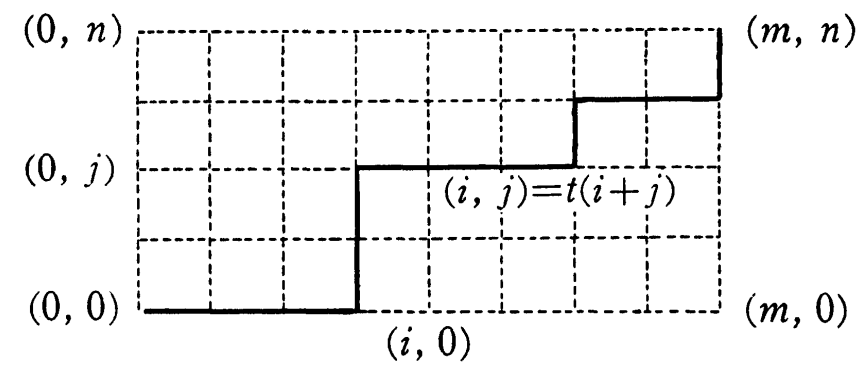

The next lemma can be seen as a generalization of the so-called prism lemma.

LEMMA 2.7. For $\tau: \Delta_{m} \times \Delta_{n} \rightarrow U(m, n \geqq 0)$,

$$
\partial C_{m, n}(\tau)=\sum_{i=0}^{m}(-1)^{i+n} C_{m-1, n}\left(\tau \circ \varepsilon_{i}^{0}\right)+\sum_{i=0}^{n}(-1)^{i} C_{m, n-1}\left(\tau \circ \varepsilon_{i}^{1}\right) .
$$

Proof. First observe

$$
\partial C_{m, n}(\tau)=\sum_{t \in O(m, n)} \sum_{k=0}^{m+n}(-1)^{c(t)+k} \tau \circ \mu_{t}{ }^{\circ} \varepsilon_{k} .
$$

If $m=0$ or $n=0$, then $C_{m, n}(\tau)=\tau$ and hence we have the formula. Next, observe that $\tau \circ \mu_{t} \circ \varepsilon_{k}$ is cancelled in the above sum if $1 \leqq k \leqq m+n-1$ and $\left(t_{\nu}(k)-t_{\nu}(k-1)\right)\left(t_{\nu}(k+1)-t_{\nu}(k)\right)=0$ for both $\nu=0,1$, i. e., $t$ is bent at the point $\left(t_{0}(k), t_{1}(k)\right)$ in the above figure. Therefore,

$$
\partial C_{m, n}(\tau)=\sum_{(t, k) \in P_{0}}(-1)^{c(t)+k} \tau^{\circ} \mu_{t^{\circ} \varepsilon_{k}}+\sum_{(t, k)) \in P_{1}}(-1)^{c(t)+k} \tau^{\circ} \mu_{t^{\circ} \varepsilon_{k},},
$$

where

$$
\begin{aligned}
P_{\nu}=\{(t, k) & \in O(m, n) \times(m+n+1): t_{\nu}(k-1)=t_{\nu}(k)=t_{\nu}(k+1) \quad \text { or } \\
k & \left.=0 \& t_{\nu}(0)=t_{\nu}(1) \quad \text { or } \quad k=m+n \& t_{\nu}(m+n+1)=t_{\nu}(m+n)\right\}
\end{aligned}
$$

for $\nu=0,1$. For $(t, k) \in P_{1}$, let $i=t_{0}(k)$ and $j=t_{1}(k)$. Then $i+j=k$. Define $t^{*} \in$ $O(m-1, n)$ by : $t^{*}(p)=t(p)$ for $p<k$, and $t^{*}(p)=\left(t_{0}(p+1)-1, t_{1}(p+1)\right)$ for $p \geqq k$. It is a routine to check $c(t)-c\left(t^{*}\right)=n-j=n+i-k$, hence $(-1)^{c(t)+k}=(-1)^{n+i+c(t *)}$. On the other hand, $\tau \circ \mu_{t} \varepsilon_{k}=\tau \circ \varepsilon_{i}^{0} \circ \mu_{t *}$. Therefore, 


$$
\begin{aligned}
\sum_{(t, k) \in P_{1}}(-1)^{c(t)+k} \tau \circ \mu_{t} \circ \varepsilon_{k} & =\sum_{i=0}^{m} \sum_{t * \in O(m-1, n)}(-1)^{i+n+c(t *)} \tau \circ \varepsilon_{i}^{0} \circ \mu_{t *} \\
& =\sum_{i=0}^{m}(-1)^{i+n} C_{m-1, n}\left(\tau \circ \varepsilon_{i}^{0}\right) .
\end{aligned}
$$

For $(t, k) \in P_{0}$, set $i$ and $j$ as above. Define $t^{*} \in O(m, n-1)$ by $: t^{*}(p)=t(p)$ for $p<k$, and $t^{*}(p)=\left(t_{0}(p+1), t_{1}(p+1)-1\right)$ for $p \geqq k$. Then, $(-1)^{k+c(t)}=(-1)^{j+c(t *)}$ and $\tau \circ \mu_{t} \circ \varepsilon_{k}=\tau \circ \varepsilon_{j}^{1} \circ \mu_{t *}$. Therefore,

$$
\begin{aligned}
\sum_{(t, k) \in P_{0}}(-1)^{c(t)+k} \tau \circ \mu_{t} \circ \varepsilon_{k} & =\sum_{j=0}^{n} \sum_{t * \in O(m, n-1)}(-1)^{j+c(t *)} \tau \circ \varepsilon_{j}^{1} \circ \mu_{t *} \\
& =\sum_{j=0}^{n}(-1)^{j} C_{m, n-1}\left(\tau \circ \varepsilon_{j}^{1}\right) .
\end{aligned}
$$

Now, we have shown the lemma.

For $s \in I(j, n+1)$, let $S_{s}=\Pi_{p=1}^{j}(-1)^{p+f_{s}^{p-1}\left(s_{p-1}\right)}$ and $S_{s}=1$ if $j=0$. Finally let $\sigma_{j}=\sum_{k=0}^{m} \lambda_{k} \sum_{s \in I(j, n+1)} S_{s} \cdot C_{n-j, j+1}\left(\tau_{s}^{k}\right)$ for $0 \leqq j \leqq n$ and $\sigma=\sum_{j=0}^{n}(-1)^{j} \cdot \sigma_{j}$. Then, $\sigma$ belongs to $S_{n+1}(U)$ by Corollary 2.6. We want to show $\partial \sigma=\sum_{j=0}^{n}(-1)^{j} \partial \sigma_{j}=$ $u-v$. By Lemma 2.7,

$$
\begin{aligned}
\partial \sigma_{j}= & \sum_{k=0}^{m} \lambda_{k} \sum_{s \in I(j, n+1)} S_{s} \cdot \partial C_{n-j, j+1}\left(\tau_{s}^{k}\right) \\
= & \sum_{k=}^{m} \lambda_{k} \sum_{s \in I(j, n+1)} S_{s} \cdot \sum_{i=0}^{n-j}(-1)^{i+j+1} C_{n-j-1, j+1}\left(\tau_{s}^{k} \circ \varepsilon_{i}^{0}\right) \\
& +\sum_{k=0}^{m} \lambda_{k} \sum_{s \in I(j, n+1)} S_{s} \cdot C_{n-j, j}\left(\tau_{s}^{k} \circ \varepsilon_{0}^{1}\right) \\
& +\sum_{k=0}^{m} \lambda_{k} \sum_{s \in I(j, n+1)} S_{s} \cdot \sum_{i=1}^{j-1}(-1)^{i} \cdot C_{n-j, j}\left(\tau_{s}^{k} \circ \varepsilon_{i}^{1}\right) \\
& +\sum_{k=0}^{m} \lambda_{k} \sum_{s \in I(j, n+1)} S_{s} \cdot \sum_{i=j}^{j+1}(-1)^{i} \cdot C_{n-j, j}\left(\tau_{s}^{k} \circ \varepsilon_{i}^{1}\right),
\end{aligned}
$$

especially,

$$
\partial \sigma_{0}=\sum_{k=0}^{m} \lambda_{k} \sum_{i=0}^{n}(-1)^{i+1} C_{n-1,1}\left(\tau_{\phi^{\circ}}^{k} \varepsilon_{i}^{0}\right)+\sum_{k=0}^{m} \lambda_{k}\left(u_{k}-v_{k}\right)
$$

LEMMA 2.8. For each $j>0$

$$
\begin{aligned}
& \sum_{k=0}^{m} \lambda_{k} \sum_{s \in I(j, n+1)} S_{s} \cdot(-1)^{j} \cdot C_{n-j, j}\left(\tau_{s}^{k} \circ \varepsilon_{j}^{1}\right)=0 \quad \text { and } \\
& \sum_{k=0}^{m} \lambda_{k} \sum_{s \in I(j, n+1)} S_{s} \cdot(-1)^{j+1} \cdot C_{n-j, j}\left(\tau_{s}^{k} \circ \varepsilon_{j+1}^{1}\right)=0
\end{aligned}
$$

Proof. For each $s \in I(j, n+1)$ and $0 \leqq k \leqq m, s^{\prime} \in I(j, n+1)$ and $0 \leqq k^{\prime} \leqq m$ are uniquely determined so that $\left(k, s_{0}\right) \sim_{u}\left(k^{\prime}, s_{0}^{\prime}\right)$ and $g\left(s_{p}\right)=s_{p}^{\prime}$ for $0<p<j$,

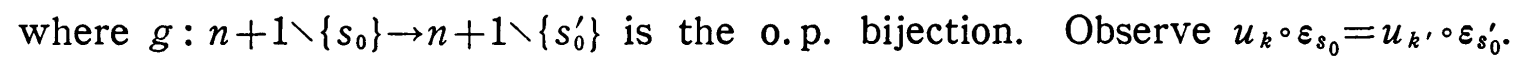
It follows $u_{k} \circ \varepsilon_{\operatorname{Im} s}=u_{k^{\prime}} \circ \varepsilon_{\operatorname{Im} s^{\prime}}$. Since $\left(k,\left\{s_{q}: 0 \leqq q<j-p\right\}\right) \sim_{u}\left(k^{\prime},\left\{s_{q}^{\prime}: 0 \leqq q<j-p\right\}\right)$ for $0 \leqq p<j, B_{s}^{k, p}=B_{s^{\prime}}^{k^{\prime}, p}$ and $F_{s}^{k, p}=F_{s}^{k^{\prime}}, p$ for $0 \leqq p<j$. By Lemma 2.5, $\tau_{s}^{k}\left(\alpha, \boldsymbol{e}_{p}\right)$ $=\tau_{s^{\prime}}^{k^{\prime}}\left(\alpha, e_{p}\right)$ for $0 \leqq p<j$ and $\tau_{s}^{k}\left(\alpha, e_{j+1}\right)=u_{k^{\circ}} \varepsilon_{\operatorname{Im} s}(\alpha)=u_{k^{\prime} \circ} \varepsilon_{\operatorname{Im} s^{\prime}}(\alpha)=\tau_{s^{\prime}}^{k^{\prime}}\left(\alpha, \boldsymbol{e}_{j+1}\right)$. Thus we have $\tau_{s}^{k} \circ \varepsilon_{j}^{1}=\tau_{s^{\prime} \circ}^{k^{\prime} \circ} \varepsilon_{j}^{1}$. By definition of $\sim_{u}, \lambda_{k}(-1)^{s_{0}}+\lambda_{k^{\prime}}(-1)^{s_{0}^{\prime}}=0$. Since $f_{s}^{0}\left(s_{0}\right)=s_{0}, f_{s^{\prime}}^{0}\left(s_{0}^{\prime}\right)=s_{0}^{\prime}$ and $f_{s}^{p-1}\left(s_{p-1}\right)=f_{s^{\prime}}^{p-1}\left(s_{p-1}^{\prime}\right)$ for $2 \leqq p<j$, we have $\lambda_{k} S_{s}+\lambda_{k^{\prime}} S_{s^{\prime}}$ $=0$. Thus we get the first equation. By replacing $\sim_{u}$ by $\sim_{v}$, we have $\tau_{s}^{k} \circ \varepsilon_{j+1}^{1}$ 
$=\tau_{s^{\prime}}^{k^{\prime} \circ} \varepsilon_{j+1}^{1}$ and $\lambda_{k} S_{s}+\lambda_{k^{\prime}} S_{s^{\prime}}=0$, which imply the second equation.

LEMMA 2.9. The following equation holds for $j>0$ and $0 \leqq k \leqq m$ :

$$
\sum_{s \in I(j, n+1)} S_{s} \cdot \sum_{p=1}^{j-1}(-1)^{p} C_{n-j, j}\left(\tau_{s}^{k} \circ \varepsilon_{p}^{1}\right)=0 .
$$

Proof. Let $0<p<j$. For each $s \in I(j, n+1), \bar{s} \in I(j, n+1)$ is uniquely determined so that $\bar{s}_{q}=s_{q}$ for $q<p-1$ or $q>p, \bar{s}_{p-1}=s_{p}$ and $\bar{s}_{p}=s_{p-1}$. Then, $B_{s}^{k, q}=$ $B_{\bar{s}}^{k, q}$ and $F_{s}^{k, q}=F_{\bar{s}}^{k, q}$ for $q \neq p$, hence $\tau_{s}^{k} \varepsilon_{p}^{1}=\tau_{\bar{s}}^{k} \circ \varepsilon_{p}^{1}$. Observe $f_{s}^{q}\left(s_{q}\right)=f_{\bar{s}}^{q}\left(\bar{s}_{q}\right)$ for $q<p-1$ or $q>p$. If $s_{p-1}<s_{p}$, then $f_{s}^{p-1}\left(s_{p-1}\right)=f_{\bar{s}}^{p}\left(\bar{s}_{p}\right)$ and $f_{s}^{p}\left(s_{p}\right)+1=f_{p}^{\bar{s}}\left(\bar{s}_{p}\right)$. Otherwise, i. e. $s_{p}<s_{p-1}, f_{\bar{s}-1}^{p}\left(\bar{s}_{p-1}\right)=f_{s}^{p}\left(s_{p}\right)$ and $f_{\bar{s}}^{p}\left(\bar{s}_{p}\right)+1=f_{s}^{p}\left(s_{p}\right)$. In any case, $S_{s}+S_{\bar{s}}=0$, which implies the lemma.

LEMMA 2.10. For $0 \leqq j<n$,

$$
\begin{gathered}
\sum_{s \in I(j, n+1)} S_{s} \cdot \sum_{i=0}^{n-j}(-1)^{i+j+1} C_{n-j-1, j+1}\left(\tau_{s}^{k} \circ \varepsilon_{i}^{0}\right) \\
=\sum_{s \in I(j+1, n+1)} S_{s} \cdot C_{n-j-1, j+1}\left(\tau_{s}^{k} \circ \varepsilon_{0}^{1}\right) .
\end{gathered}
$$

Proof. For each $s \in I(j, n+1)$ and $i \leqq n-j, s_{*} \in I(j+1, n+1)$ corresponds uniquely to the pair $(s, i)$ so that $s_{*}(p)=s(p)$ for $p<j$ and $s_{*}(j)=g(i)$, where $g: n+1-j \rightarrow n+1 \backslash\left\{s_{p}: 0 \leqq p<j\right\}$ is the o.p. bijection. Then $f_{s *}^{j}=g^{-1}$ and $s_{* j}=$ $g(i)$. By the definition of $\tau_{s *}^{k}, \tau_{s *}^{k}{ }^{\circ} \varepsilon_{0}^{1}\left(\alpha, \boldsymbol{e}_{p}\right)=\tau_{s^{\circ}}^{k} \varepsilon_{f_{s *}^{0}}^{i}\left(s_{* j}\right)\left(\alpha, \boldsymbol{e}_{p}\right)=\tau_{s}^{k} \varepsilon_{i}^{0}\left(\alpha, \boldsymbol{e}_{p}\right)$ for each $0 \leqq p \leqq j . \quad$ Since $f_{s *}^{p-1}\left(s_{* p-1}\right)=f_{s}^{p-1}\left(s_{p-1}\right)$ for $1 \leqq p \leqq j$ and $f_{s_{*}}^{j}\left(s_{* j}\right)=i, \quad S_{s_{*}}=$ $(-1)^{i+j+1} S_{s}$. Therefore,

$$
\begin{gathered}
(-1)^{i+j+1} S_{s} \cdot C_{n-j-1, j+1}\left(\tau_{s}^{k} \circ \varepsilon_{i}^{0}\right)=S_{s *} \cdot C_{n-j-1, j+1}\left(\tau_{s *}^{k} \circ \varepsilon_{0}^{1}\right) \quad \text { and } \\
(-1)^{j+1} \sum_{s \in I(j, n+1)} S_{s} \cdot \sum_{i=0}^{n-j}(-1)^{i} C_{n-j-1, j+1}\left(\tau_{s}^{k} \circ \varepsilon_{i}^{0}\right) \\
=\sum_{s \in I(j+1, n+1)} S_{s} \cdot C_{n-j-1, j+1}\left(\tau_{s}^{k} \circ \varepsilon_{0}^{1}\right) .
\end{gathered}
$$

Recalling the formula before Lemma 2.8, we have

$$
\begin{aligned}
\partial \sigma= & \sum_{j=0}^{n}(-1)^{j} \partial \sigma_{j} \\
= & \sum_{k=0}^{m} \lambda_{k}\left(u_{k}-v_{k}\right) \\
& +(-1)^{n} \sum_{k=0}^{m} \lambda_{k} \sum_{s \in I(n, n+1)} S_{s} \cdot \sum_{i=0}^{0}(-1)^{i+n+1} C_{-1, n+1}\left(\tau_{s}^{k} \circ \varepsilon_{i}^{0}\right) \\
= & \sum_{k=0}^{m} \lambda_{k}\left(u_{k}-v_{k}\right)=u-v .
\end{aligned}
$$

by Lemmas 2.8, 2.9 and 2.10 ,

Now, we have completed the proof of Theorem 2.1. 


\section{Broken path connectedness and quasi-homotopy}

Let $x, y \in X$. We write $\underset{p}{\sim} y$ if $x$ and $y$ are connected by a path, i.e., there is a continuous map $f: \boldsymbol{I} \rightarrow X$ such that $f(0)=x$ and $f(1)=y$. A finite sequence of continuous maps $f_{i}: I \rightarrow X(i=0, \cdots, n)$ are called a broken path. And we say that $\left(f_{i}\right)_{i=0}^{n}$ connects $x$ to $y$ if $f_{0}(0)=x$ and $f_{n}(1)=y$. For $\rho \in$ $\operatorname{Pm}(X)$, the $\rho$-gap of a broken path $\left(f_{i}\right)_{i=0}^{n}$ in $X$ is $\sum_{i=0}^{n-1} \rho\left(f_{i}(1), f_{i+1}(0)\right)$. In case $n=0$, the $\rho$-gap is 0 . We write $\underset{b}{\sim} y$ if for any $\rho \in \operatorname{Pm}(X)$ and $\varepsilon>0, x$ and $y$ are connected by a broken path with the $\rho$-gap less than $\varepsilon$, i.e., there are $x_{i}, y_{i} \in X(i=0, \cdots, n)$ such that $x_{0}=x, y_{n}=y, \underset{p}{x_{i} \sim y_{i}}$ for each $i=0, \cdots, n$ and $\sum_{i=0}^{n-1} \rho\left(y_{i}, x_{i+1}\right)<\varepsilon$. Then $\underset{b}{\sim}$ is an equivalence relation on $X$. It is said that $X$ is broken path connected if $\underset{b}{\sim} y$ for any $x, y \in X$.

Proposition 3.1. Let $x, y \in X$, then $x \underset{b}{\sim} y$ iff $f(x)=f(y)$ for any continuous map $f: X \rightarrow \boldsymbol{R}$ which is constant on each path component.

Proof. For an arbitrary continuous map $f: X \rightarrow \boldsymbol{R}$ which is constant on each path component, define $\rho \in \operatorname{Pm}(X)$ by: $\rho(u, v)=|f(u)-f(v)|$. Since $u \underset{p}{\sim} v$ implies $\rho(u, v)=0, \underset{b}{\underset{b}{\sim} y}$ implies $\rho(x, y)=0$, that is, $f(x)=f(y)$. To see the converse implication, for any $\rho \in \operatorname{Pm}(X)$ we define $f: X \rightarrow \boldsymbol{R}$ by :

$$
f(u)=\inf \left\{\sum_{i=0}^{n-1} \rho\left(u_{i}, x_{i+1}\right): x_{i} \underset{p}{\sim} u_{i}(0 \leqq i \leqq n), x_{0}=x, u_{n}=u\right\} .
$$

Then, $f$ is continuous and $f(u)=f(v)$ if $\underset{p}{u \sim v}$. From the assumption, $f(y)=$ $f(x)=0$, which implies $x \underset{b}{\sim} y$.

Let $X=\left\{(x, y) \mid y=0\right.$ or $\left.y=x^{-1}\right\} \subset \boldsymbol{R}^{2}$ and $\rho$ be the Euclidean metric on $X$. Then each pair of points of $X$ are connected by broken paths with arbitrarily small $\rho$-gaps. However $X$ is not broken path connected because $X$ is homeomorphic to three parallel straight lines in the plane. The following is such an example in case $X$ is compact.

EXAmple 3.2. Concerning Cantor's ternary set, the following is well-known. There exist families $\left\{I_{i}: i \in \boldsymbol{N}\right\}$ and $\left\{J_{i}: i \in \boldsymbol{N}\right\}$ of pairwise disjoint open subintervals of / such that $\mu\left(\cup_{i \in N} I_{i}\right)=1, \mu\left(\cup_{i \in N} J_{i}\right)=1 / 2$ and both $I \backslash \cup_{i \in N} I_{i}$ and $I \backslash \cup_{i \in N} J_{i}$ are nowhere-dense perfect sets, where $\mu$ is the Lebesque meaure. Then there exists a homeomorphism $h: I \rightarrow I$ such that $h\left(\cup_{i \in N} I_{i}\right)=\cup_{i \in N} J_{i}$. 
Let

$$
\begin{aligned}
W= & \{(x, y): y=\sin (\pi / x), 0<x \leqq 1 / 2\} \cup \\
& \{(x,-y): y=\sin (\pi /(1-x)), 1 / 2<x<1\} .
\end{aligned}
$$

For each $i \in N$, let $h_{i}:(0,1) \rightarrow I_{i}$ be a homeomorphism and $W_{i}=\left(h_{i} \times \mathrm{id}\right)(W)$. We define $X=\left(\boldsymbol{I} \backslash \cup_{i \in N} I_{i}\right) \times[-1,1] \cup \cup_{i \in N} W_{i}$. Then, $X$ is a plane compactum. Any path in $X$ is included in $\{\alpha\} \times[-1,1]$ for some $\alpha \in I$ or $W_{i}$ for some $i \in N$. Since $\mu\left(\cup_{i \in N} I_{i}\right)=1$, the infimum of $\rho$-gaps of broken paths connecting $(0,0)$ to $(1,0)$ in $X$ is equal to 0 , where $\rho$ is the Euclidean metric on the plane. On the other hand, the infimum of $\rho$-gaps of broken paths connecting $(0,1)$ to $(1,0)$ in $(h \times \mathrm{id})(X)$ is $1 / 2$ by the same reason. Therefore, $X$ is not broken path connected.

Any path connected space is obviously broken path connected but the converse does not hold. The example has been given in Example 3.2, i. e., $W \cup$ $\{0,1\} \times[-1,1]$ is broken path connected but not path connected. Any broken path connected space is connected but the converse does not hold. In fact, the space in Example 3.2 is such an example. The pseudo-arc $\boldsymbol{P}$ is also such a continuum since it has no nontrivial paths.

One should remark that each equivalence class of $\underset{b}{\sim}$ is closed in $X$ which contains a path component but it need not be connected. For example, let

$$
X=\cup_{n=1}^{\infty}\{1 / n\} \times I \cup\{(0,0),(0,1)\} .
$$

Then $A=\{(0,0),(0,1)\}$ is an equivalence class of $\underset{b}{\sim}$. In case $X$ is compact, it is connected as shown in the next proposition. But even if $X$ is compact metric, it need not be broken path connected. For example, let $\boldsymbol{P}$ be a pseudo-arc in the plane and let $A_{n}(n \in \boldsymbol{N})$ be a sequence of arcs which converges to $\boldsymbol{P}$ in the hyperspace. Define $X=\cup_{n=1}^{\infty}\{1 / n\} \times A_{n} \cup\{0\} \times \boldsymbol{P} \subset \boldsymbol{R}^{3}$. Then, $X$ is compact metrizable and $\{0\} \times \boldsymbol{P}$ is an equivalence class of $\underset{b}{ }$.

Proposition 3.3. If $X$ is compact, then each equivalence class of $\sim_{b}$ is connected.

Proof. For each $\rho \in \operatorname{Pm}(X)$, take a broken path from $x$ to $y$ with the $\rho$ gap less than 1 and let $K_{\rho}$ be the union of the images of its paths. For $\rho, \rho^{\prime}$ $\in \operatorname{Pm}(X)$, define $\rho \leqq \rho^{\prime}$ by $: \rho(x, y) \leqq \rho^{\prime}(x, y)$ for all $x, y \in X$. Then $(\operatorname{Pm}(X), \leqq)$ is a directed set. Since the hyperspace $2^{X}$ with the Vietoris topology is compact, the net $\left(K_{\rho}: \rho \in \operatorname{Pm}(X)\right)$ has a cluster point $K \in 2^{X}$. Clearly, $K$ con- 
tains both $x$ and $y$. Suppose that $K$ is a disjoint union of nonempty closed subsets $A$ and $B$. Take $\rho \in \operatorname{Pm}(X)$ so that $\rho(a, b)>3$ for each $a \in A$ and $b \in B$. Let $U=\{x \in X: \rho(x, A)<1\}$ and $V=\{x \in X: \rho(x, B)<1\}$. Then there exists $\rho^{\prime} \in \operatorname{Pm}(X)$ such that $\rho \leqq \rho^{\prime}, K_{\rho^{\prime}} \subset U \cup V, K_{\rho^{\prime}} \cap U \neq \varnothing$ and $K_{\rho^{\prime}} \cap V \neq \varnothing$. This contradicts to the fact that the $\rho^{\prime}$-gap of the broken path is less than 1 .

Now, we prove the following.

THEOREM 3.4. For any space $X, H_{0}^{T}(X)$ is canonically isomorphic to the free abelian group whose rank is equal to the cardinality of equivalence classes with respect to $\underset{b}{\sim}$.

COROLLARY 3.5. A space $X$ is broken path connected if and only if $H_{0}^{T}(X)$ $=Z$.

To show the theorem we must recall the topology of the free abelian topological group $A(X)$, because $Z_{0}(X)=S_{0}(X)$ is identical with $A(X)$. Any $\rho \in$ $\operatorname{Pm}(X)$ can be extended to $\tilde{\rho} \in \operatorname{Pm}(A(X))$ by the same way as metrics [12] (cf. Section 2). We abuse $\rho$ with $\tilde{\rho}$ as before. It is known that the topology of the free abelian topological group $A(X)$ is determined by all such pseudo-metrics $\rho[20$, p. 379 or 24 , Theorem 1]. Here, we outline the proof. By [16], the topology of $A(X)$ is determined by all invariant continuous pseudo-metrics $\tau$ on $A(X)$. Let $\rho \in \operatorname{Pm}(A(X))$ be the extension of $\tau \mid X$ in the above manner. Since $\tau(a+b, c+d) \leqq \tau(a, c)+\tau(b, d)$ by the invariantness of $\tau$,

$$
\{a \in A(X): \rho(a, 0)<\varepsilon\} \subset\{a \in A(X): \tau(a, 0)<\varepsilon\} \quad \text { for } \varepsilon>0 .
$$

Thus we get the conclusion. The theorem is an immediate consequence of the next lemma, where $C\left(\Delta_{0}, X\right)$ is naturally identified with $X$.

LEMMA 3.6. $\overline{B_{0}(X)}=\overline{\langle x-y: x \underset{p}{x} y\rangle}=\langle x-y: x \underset{b}{\sim} y\rangle$ in $A(X)$.

Proof. Suppose that $\underset{b}{\sim} y$. For each $\rho \in \operatorname{Pm}(X)$ and $\varepsilon>0$, we have $x_{i}, y_{i}$ $\in X(0 \leqq i \leqq n)$ such that $x_{i} \underset{p}{\sim} y_{i}, x_{0}=x, y_{n}=y$ and $\sum_{i=0}^{n-1} \rho\left(y_{i}, x_{i+1}\right)<\varepsilon$. Then, $\sum_{i=0}^{n}\left(x_{i}-y_{i}\right) \in B_{0}$ and

$$
\begin{aligned}
\rho\left(x-y, \sum_{i=0}^{n}\left(x_{i}-y_{i}\right)\right) & =\rho\left(0, \sum_{i=0}^{n} x_{i}-\sum_{i=0}^{n} y_{i}-x+y\right) \\
& =\rho\left(0, \sum_{i=1}^{n} x_{i}-\sum_{i=0}^{n-1} y_{i}\right) \\
& \leqq \sum_{i=0}^{n-1} \rho\left(y_{i}, x_{i+1}\right)<\varepsilon .
\end{aligned}
$$


Hence $x-y \in \overline{B_{0}}$.

To see the other inclusion, we introduce some notion and notation. For each $u \in X \cup(-X),|u|=u$ if $u \in X$ and $|u|=-u$ if $u \in-X$. We identify $-(-x)$ with $x$ for $x \in X$. A reduced form of a non-zero $u \in A(X)$ is $u=\sum_{i=1}^{n} u_{i}$, where $u_{i} \in X \cup(-X)(1 \leqq i \leqq m)$ and $u_{i} \neq-u_{j}$ for any $i, j$. Let $u \in \overline{B_{0}}(u \neq 0)$ and $u=$ $\sum_{i=1}^{m} u_{i}$ be a reduced form of $u$. We claim that there exist $u_{i} \in X$ and $u_{j} \in-X$ such that $u_{i} \underset{b}{\sim}-u_{j}$. Suppose the contrary. Then there exist $\rho \in \operatorname{Pm}(X)$ and $0<\varepsilon<1$ with the following: $\rho(u, 0)>\varepsilon ; u_{i} \in X$ and $u_{j} \in-X$ imply that the $\rho$ gap of any broken path connecting $u_{i}$ to $-u_{j}$ is greater than $\varepsilon$. Since $u \in \overline{B_{0}}$, there exist $x_{i}, y_{i} \in X(0 \leqq i \leqq n)$ such that $x_{i} \underset{p}{\sim} y_{i}(0 \leqq i \leqq n)$ and $\rho\left(u, \sum_{i=0}^{n}\left(x_{i}-y_{i}\right)\right)$ $<\varepsilon$. We may assume that $\sum_{i=0}^{n}\left(x_{i}-y_{i}\right)$ is a reduced form. Since $u \neq 0$ and $\rho\left(u, \sum_{i=0}^{n}\left(x_{i}-y_{i}\right)\right)<1$, there exist $a_{i}, b_{i} \in X \cup(-X)(1 \leqq i \leqq k)$ such that $u=\sum_{i=1}^{k} a_{i}$, $\sum_{i=0}^{n}\left(x_{i}-y_{i}\right)=\sum_{i=1}^{k} b_{i} \quad$ and $\rho\left(u, \sum_{i=0}^{n}\left(x_{i}-y_{i}\right)\right)=\sum_{i=1}^{k} \rho\left(a_{i}, b_{i}\right)$ [12]. Since $\sum_{i=0}^{n}\left(x_{i}-y_{i}\right)=\sum_{i=1}^{k} b_{i}, k$ is even. Since $\sum_{i=1}^{m} u_{i}=\sum_{i=1}^{k} a_{i}, m$ is also even. We may assume that $u_{1} \in X, a_{i}=u_{i}$ for $i=1, \cdots, m$ and moreover $a_{m+2 i-1}=-a_{m+2 i} \in X$ for $i=1, \cdots,(k-m) / 2$. We can choose a partion $P$ of $\{1, \cdots, k\}$ such that each element of $P$ has exactly two elements and $\{i, j\} \in P$ implies that $b_{i} \in X$ iff $b_{j} \in-X$, and $\left|b_{i}\right|{\underset{p}{p}}_{b_{j}} \mid$. (Note that $b_{i}=-b_{j}$ implies $\left|b_{i}\right| \underset{p}{\sim_{p}}\left|b_{j}\right|$ ) We get a sequence

$$
a_{i_{1}}, b_{i_{1}}, b_{i_{2}}, a_{i_{2}}, a_{i_{3}}, b_{i_{3}}, \cdots, b_{i_{2 h}}, a_{i_{2 h}}
$$

such that $i_{1}=1, i_{2 h} \leqq m,\left\{i_{2 j-1}, i_{2 j}\right\} \in P$ for $j=1, \cdots, h$ and $i_{2 j+1}=i_{2 j}-1>m$ for $j=1, \cdots, h-1$. Then $b_{i_{2 j-1}} \in X, b_{i_{2 i-1}} \underset{p}{\sim}-b_{i_{2 j}}$ for $j=1, \cdots, h$ and $a_{i_{2 j+1}}=-a_{i_{2 j}}$ $\in X$ for $j=1, \cdots, h-1$. Then,

$$
\begin{aligned}
& \rho\left(\left|a_{i_{1}}\right|,\left|b_{i_{1}}\right|\right)+\sum_{j=1}^{h-1} \rho\left(\left|b_{i_{2 j}}\right|,\left|b_{i_{2 j+1}}\right|\right)+\rho\left(\left|b_{i_{2 h}}\right|,\left|a_{i_{2 h}}\right|\right) \\
\leqq & \rho\left(\left|a_{i_{1}}\right|,\left|b_{i_{1}}\right|\right)+\sum_{j=1}^{h-1}\left(\rho\left(\left|b_{i_{2 j}}\right|,\left|a_{i_{2 j}}\right|\right)+\rho\left(\left|a_{i_{2 j+1}}\right|,\left|b_{i_{2 j+1}}\right|\right)\right) \\
& +\rho\left(\left|b_{i_{2 h}}\right|,\left|a_{i_{2 h}}\right|\right) \\
= & \sum_{j=1}^{2 h} \rho\left(\left|a_{i_{j}}\right|,\left|b_{i_{j}}\right|\right) \\
\leqq & \sum_{i=1}^{k} \rho\left(\left|a_{i}\right|,\left|b_{i}\right|\right)<\varepsilon,
\end{aligned}
$$

that is, we have a broken path connecting $u_{1}=a_{i_{1}}$ to $u_{i_{2 h}}=a_{i_{2 h}}$ with the $\rho$-gap less than $\varepsilon$. This contradicts to the hypothesis. Now, we have shown the claim, from which lemma follows by induction.

Next we define a notion "quasi-homotopy". For $f, g \in C(X, Y)$, we write $f \widetilde{h} g$ if $f$ and $g$ are homotopic. In case $X$ is locally compact, as is well-known 
$f \widetilde{h} g$ iff $f \underset{p}{\sim} g$, where $C(X, Y)$ is endowed with compact-open topology. We say $f$ and $g$ are quasi-homotopic (denoted by $f \sim_{q} g$ ) if the statement obtained by replacing $\underset{p}{\sim}$ and $X$ by $\underset{n}{\sim}$ and $C(X, Y)$ in the definition of broken path connectedness. More precisely, $f \widetilde{q}_{g} g$ if for any $\rho \in \operatorname{Pm}(C(X, Y))$ and $\varepsilon>0$ there exist $f_{i}, g_{i}(0 \leqq i \leqq n)$ such that $f_{0}=f, g_{n}=g, f_{i} \sim_{h} g_{i}$ and $\sum_{i=0}^{n-1} \rho\left(g_{i}, f_{i+1}\right)<\varepsilon$. In case $X$ is locally compact, $f \sim_{q} g$ iff $f \widetilde{b}_{g}$.

Analogously to Proposition 3.1 and Lemma 3.5, we get

Proposition 3.7. Let $C(X, Y)$ be endowed with compact-open topology and $f, g \in C(X, Y)$. Then, $f \underset{q}{\sim} g$ iff $F(f)=F(g)$ for any continuous map $F: C(X, Y)$ $\rightarrow \boldsymbol{R}$ such that $F(u)=F(v)$ when $u_{\tilde{n}} v$.

Proposition 3.8. Let $\mathscr{H}(X, Y)=\langle f-g: f \widetilde{n} g, f, g \in C(X, Y)\rangle$ be the subgroup of the free abelian topological group $A(C(X, Y))$ over $C(X, Y)$. Then, $f \underset{q}{\sim} g$ iff $f-g \in \overline{\mathcal{H}(X, Y)}$.

THEOREM 3.9. For $f, g \in C(X, Y)$, if $f$ and $g$ are quasi-homotopic, then $f_{*}^{T}=g_{*}^{T}$ holds.

Proof. Let $\rho \in \operatorname{Pm}\left(C\left(\Delta_{n}, Y\right)\right)$. For $z \in Z_{n}(X)$, define $\rho^{\prime} \in \operatorname{Pm}(C(X, Y))$ by : $\rho^{\prime}(f, g)=\rho\left(f_{\#} z, g_{\#} z\right)$. For each $\varepsilon>0$, there exist $f_{i}, g_{i} \in C(X, Y)(0 \leqq i \leqq m)$ such that $f_{0}=f, g_{m}=g, f_{i} \sim_{n} g_{i}$ and $\sum_{i=0}^{n-1} \rho^{\prime}\left(f_{i+1}, g_{i}\right)<\varepsilon$, which implies

$$
\begin{aligned}
\rho\left(f_{\#} z-g_{\#} z, \sum_{i=0}^{n}\left(f_{i \#} z-g_{i \#} z\right)\right) & \leqq \sum_{i=0}^{n-1} \rho\left(f_{i+1 \#} z, g_{i \#} z\right) \\
& =\sum_{i=1}^{n-1} \rho^{\prime}\left(f_{i+1}, g_{i}\right)<\varepsilon .
\end{aligned}
$$

Hence $f_{\#} z-g_{\#} z \in \overline{B_{n}(Y)}$.

COROLlary 3.10. If there exist continuous maps $f: X \rightarrow Y$ and $g: Y \rightarrow X$ such that $g \circ f \underset{q}{\sim} \mathrm{id}_{X}$ and $f \circ g \underset{q}{\sim} \operatorname{id}_{Y}$, then $H_{n}^{T}(X) \cong H_{n}^{T}(Y)$.

Here we give some maps which are quasi-homotopic.

EXAMPLE 3.11. (1) Let

$$
\begin{gathered}
X=\left\{(x, y, 0):(x-1)^{2}+y^{2}=1\right\} \cup\left\{(x, y, 1 / m):(x-1)^{2}+y^{2} \leqq 1, n \in N\right\} \\
\cup\{(0,0, z): 0 \leqq z \leqq 1\}
\end{gathered}
$$


and define $f_{m}: X \rightarrow X$ by : $f_{m}(x, y, z)= \begin{cases}(x, y, z) & \text { if } z \geqq 1 / m, \\ (x, y, 1 / m) & \text { otherwise. }\end{cases}$

Then, $\mathrm{id} \underset{q}{f_{1}} f_{1}$, since any neighborhood of $i d_{X}$ contains some $f_{m}$ and $f_{m} \widetilde{f}_{h} f_{1}$. Consequently $H_{n}^{T}(X)=0$.

(2) Let

$$
\begin{aligned}
X= & \left\{(x, y, z):(x-\cos 2 \pi \theta)^{2}+y^{2}+(z-\sin 2 \pi \theta)^{2} \leqq 1: \theta \in \boldsymbol{Q}\right\} \\
& \cup\left\{(x, y, z):(x-\cos 2 \pi \theta)^{2}+y^{2}+(z-\sin 2 \pi \theta)^{2}=1: \theta+\sqrt{2} \in \boldsymbol{Q}\right\} .
\end{aligned}
$$

Then, similarly as the previous example, $\mathrm{id}_{X}$ is quasi-homotopic to a constant map and consequently $H_{n}^{T}(X)=0$.

\section{4. $H_{n}^{T}(X)$ for products and sums}

Let $X_{i}(i \in I)$ be spaces with base points $a_{i}$. For an element $u$ of the direct product $\Pi_{i \in I} X_{i}$, the support of $u$ is the set supp $u=\left\{i \in I: u(i) \neq a_{i}\right\}$. The $\Sigma$ product $\tilde{\Pi}_{i \in I} X_{i}$ denotes the subspaces of $\Pi_{i \in I} X_{i}$ consisting of all $u$ with countable supports. Let $\widetilde{\nabla}_{i \in I} X_{i}=\left\{u \in \Pi_{i \in I} X_{i}\right.$ : supp $u$ is at most one $\}$ denote the subspace of $\Pi_{i \in I} X_{i}$ with base point $a=\left(a_{i}\right)_{i \in I}$. By $\vee_{i \in I} X_{i}$, we denote the quotient space of the discrete sum of $X_{i}$ by identifying all $a_{i}$ 's, where the identified point $a$ is the base point. These spaces $\tilde{V}_{i \in I} X_{i}$ and $\vee_{i \in I} X_{i}$ can be regarded as spaces with the same underlying set. In case each $X_{i}$ includes a copy $A_{i}$ of $A, \vee_{i \in I}\left(X_{i}, A_{i}\right)$ is the quotient space of the discrete sum of $X_{i}$ by identifying all $A_{i}$ 's, which generalizes the one point case.

Corresponding to the above, for groups $C_{i}(I \in I), \tilde{\Pi}_{i \in I} C_{i}$ denotes the subgroup of the direct product $\prod_{i \in I} C_{i}$ consisting of all $u$ with countable supports, i. e., supp $u=\{i \in I: u(i) \neq 0\}$ is countable. The direct sum of $C_{i}$ is denoted by $\bigoplus_{i \in I} C_{i}$, i.e., $\bigoplus_{i \in I} C_{i}=\left\{u \in \Pi_{i \in I} C_{i}:\right.$ supp $u$ is finite $\}$. In case $C_{i} \cong C$ for all $i \in I, \Pi_{i \in I} C_{i}, \tilde{\Pi}_{i \in I} C_{i}$ and $\oplus_{i \in I} C_{i}$ are abbreviated by $C^{I}, \tilde{\Pi}_{I} C$ and $\oplus_{I} C$ respectively.

In general $H_{1}$ does not commute with direct products for path connected spaces, but $H_{1}^{T}$ does, that is,

Proposition 4.1. Let $X_{i}(i \in I)$ be $(n-1)$-connected spaces. Then $H_{n}^{T}\left(\Pi_{i \in I} X_{i}\right)$ $\cong \Pi_{i \in I} H_{n}^{T}\left(X_{i}\right)$ and $H_{n}^{T}\left(\tilde{\Pi}_{i \in I} X_{i}\right) \cong \tilde{\Pi}_{i \in I} H_{\pi l}^{T}\left(X_{i}\right)$ canonically for $n \geqq 1$.

We need the following lemma.

LEMMA 4.2. Let $X$ and $Y$ be (n-1)-connected spaces, $z \in Z_{n}(X \times Y)$ and $p_{X}: X \times Y \rightarrow X, p_{Y}: X \times Y \rightarrow Y$ be the projections. If $p_{X \#} z \in \overline{B_{n}(X)}$ and $p_{Y \#} z \in$ 
$\overline{B_{n}(Y)}$, then $z \in \overline{B_{n}(X \times Y)}$.

ProOF. Note that $X \times Y$ is $(n-1)$-connected. By Hurewicz's theorem [23, Ch. 7, §5], we may assume the following: In case $n$ is odd, $z \in C\left(\Delta_{n}, X \times Y\right)$ and $\operatorname{Im} z \circ \varepsilon_{i}=\left\{\left(x_{0}, y_{0}\right)\right\}$ for $0 \leqq i \leqq n$; In case $n$ is even, $z=u-v$ for $u, v \in$ $C\left(\Delta_{n}, X \times Y\right)$ and $\operatorname{Im} u \circ \varepsilon_{i}=\operatorname{Im} v=\left\{\left(x_{0}, y_{0}\right)\right\}$ for $0 \leqq i \leqq n$.

Since the proofs are not so different, we prove only in case $n$ is odd. Define $z_{X}(\alpha)=\left(p_{X} \circ z(\alpha), y_{0}\right), z_{Y}(\alpha)=\left(x_{0}, p_{Y} \circ z(\alpha)\right)$ for $\alpha \in \Delta_{n}$. Then, $z-\left(z_{X}+z_{Y}\right) \in$ $B_{n}(X \times Y)$. Hence, it suffices to show $z_{X}+z_{Y} \in \overline{B_{n}(X \times Y)}$. By the assumption, $\left.z_{X} \in \overline{B_{n}\left(X \times\left\{y_{0}\right\}\right.}\right)$ and $z_{Y} \in \overline{B_{n}\left(\left\{x_{0}\right\} \times Y\right)}$, where the closures are taken in $S_{n}\left(X \times\left\{y_{0}\right\}\right)$ and $S_{n}\left(\left\{x_{0}\right\} \times Y\right)$ respectively. Since $C\left(\Delta_{n}, X \times\left\{y_{0}\right\}\right)$ and $C\left(\Delta_{n},\left\{x_{0}\right\} \times Y\right)$ are retracts of $C\left(\Delta_{n}, X \times Y\right)$, the closures can be regarded as the ones in $S_{n}(X \times Y)$. Hence $z_{X}+z_{Y} \in \overline{B_{n}(X \times Y)}$.

Proof of Proposition 4.1. Let $p_{i}: \Pi_{i \in I} X_{i} \rightarrow X_{i}$ be the projections and define $\varphi: H_{n}^{T}\left(\Pi_{i \in I} X_{i}\right) \rightarrow \prod_{i \in I} H_{n}^{T}\left(X_{i}\right)$ by $\varphi(a)(i)=p_{i *}^{T}(a)$. By using Hurewicz's theorem, it is easy to see that $\varphi$ is an epimorphism. It suffices to show $\operatorname{Ker} \varphi$ $=\bigcap_{i \in I} \operatorname{Ker} p_{i *}^{T}=0$.

Let $z=\sum_{k=0}^{m} \lambda_{k} u_{k} \in Z_{n}\left(\lambda_{k}= \pm 1, u_{k} \in C\left(\Delta_{n}, \Pi_{i \in I} X_{i}\right)\right)$ such that $z+\overline{B_{n}} \in \operatorname{Ker} \varphi$, i.e., $p_{i \#}(z) \in \overline{B_{n}\left(X_{i}\right)}$ for each $i \in I$. For any open neighborhood $U$ of $z$ in $S_{n}\left(\Pi_{i \in I} X_{i}\right)$, there exist compact subsets $K_{k}^{j}$ of $\Delta_{n}$ and basic open sets $U_{k}^{j}$ of $\Pi_{i \in I} X_{i}(0 \leqq j \leqq r)$ such that $u_{k} \in \bigcap_{j=0}^{r} O\left(K_{k}^{j}, U_{k}^{j}\right)$ and $\sum_{k=0}^{m} \lambda_{k}\left(\bigcap_{j=0}^{r} O\left(K_{k}^{j}, U_{k}^{j}\right)\right) \subset U$, where $O(K, U)=\left\{u \in C\left(\Delta_{n}, \Pi_{i \in I} X_{i}\right): u(K) \subset U\right\}$. There exists a finite subset $F$ of $I$ such that $U_{k}^{j}$, s only depend on $F$. Define $u_{k}^{\prime} \in C\left(\Delta_{n}, \Pi_{i \in F} X_{i}\right)$ by : $p_{i \#}\left(u_{k}^{\prime}\right)=$ $p_{i \#}\left(u_{k}\right)$ for $i \in F$. Since each $X_{i}$ is $(n-1)$-connected, $\left.\sum_{k=0}^{m} \lambda_{k} u_{k}^{\prime} \in \overline{B_{n}\left(\Pi_{i \in F} X_{i}\right.}\right)$ by Lemma 4.2. Pick an element $x_{i} \in X_{i}$ for each $i \in I$ and define $u_{k}^{\prime \prime} \in C\left(\Delta_{n}, \Pi_{i \in I} X_{i}\right)$ as follows : $u_{k}^{\prime \prime}(i)=u_{k}^{\prime}(i)$ for $i \in F$ and $u_{k}^{\prime \prime}(i)=x_{i}$ for $i \notin F$. Then, $u_{k}^{\prime \prime} \in \bigcap_{j=0}^{r} O\left(K_{k}^{j}, U_{k}^{j}\right)$ hence $\sum_{k=0}^{m} \lambda_{k} u_{k}^{\prime \prime} \in U \cap B_{n}$. Therefore $z \in \overline{B_{n}}$ and $\operatorname{Ker} \varphi=0$. By separability of $\Delta_{n} \varphi\left(H_{n}^{T}\left(\tilde{\Pi}_{i \in I} X_{i}\right)\right) \subset \tilde{\Pi}_{i \in I} H_{n}^{T}\left(X_{i}\right)$. Therefore, the proof for $\Sigma$-products can be done similarly.

To calculate $H_{n}^{T}(X)$ for attaching spaces $X$, we introduce properties $\left(*_{n}\right)$ and $(* *)$ for $(X, A)$. These properties are necessary only for $n \geqq 2$.

$\left(*_{n}\right)$ If $\sum_{k=0}^{m} \lambda_{k} u_{k} \in Z_{n} \quad\left(\lambda_{k} \in Z, u_{k} \in C\left(\Delta_{n}, X\right)\right)$ and $U_{k}$ is an open neighborhood of $u_{k}$, then there exist $v_{k} \in U_{k}(0 \leqq k \leqq m)$ and $Y \subset X$ such that $A \subset Y$, Im $v_{k}$ $\subset Y, \sum_{k=0}^{m} \lambda_{k} v_{k} \in Z_{n}$ and some neighborhood of $A$ in $Y$ deforms into $A$ in $Y$. 
(**) For any open neighborhood $O$ of $\mathrm{id} \in C(X, X)$ with the compact-open topology, there exist $Y \subset X$ and $f \in O$, such that $A \subset Y, f(X) \subset Y, f \mid A=$ id and some neighborhood of $A$ in $Y$ deforms into $A$ in $Y$.

It can be clearly seen that $(* *)$ implies $\left(*_{n}\right)$ for every $n$.

LEMmA 4.3. If $\left(X_{i}, A\right)$ satisfies the property (**) and $A$ is closed in $X_{i}$ for each $i \in I$, then $\left(\bigvee_{i \in I}\left(X_{i}, A\right), A\right)$ satisfies (**). In case $A=\{a\},\left(\tilde{\nabla}_{i \in I} X_{i}, a\right)$ also satisfies $(* *)$.

Proof. Let $K_{j} \subset \bigvee_{i \in I}\left(X_{i}, A\right)$ be compact and $U_{j}$ open neighborhoods of $K_{j}$ $(0 \leqq j \leqq m)$. Then there exists a finite subset $F$ of $I$ such that $K_{j} \subset \vee_{i \in F}\left(X_{i}, A\right)$ for every $0 \leqq j \leqq m$. There exist $f_{i}: X_{i} \rightarrow Y_{i}(i \in F)$ so that $f_{i}\left(K_{j} \cap X_{i}\right) \subset U_{j} \cap X_{i}$ with other properties in (**). Since $A$ is closed, we have a continuous map $f: \bigvee_{i \in F} X_{i} \rightarrow \bigvee_{i \in F} Y_{i}$ with $f \mid X_{i}=f_{i}$ for each $i \in I$. Then $f$ satisfies the desired properties.

In case of $\bigvee_{i \in I} X_{i}$, we may assume that each $U_{j}$ is a basic open subset for each $j$. Therefore, there exists a finite subset $F$ of $I$ such that every $U_{j}$ only depends on $\bigvee_{i \in F} X_{i}$. Thus the proof is same as the above.

THEOREM 4.4. Let $X$ and $Y$ be spaces such that $X \cap Y(=A)$ is an acyclic retract of both $X$ and $Y$.

(Case $n=1) \quad H_{1}^{T}\left(X \bigcup_{A} Y\right) \cong H_{1}^{T}(X) \oplus H_{1}^{T}(Y)$ canonically.

(Case $n \geqq 2)$ If $X$ and $Y$ are normal and $\left(X \bigcup_{A} Y, A\right)$ satisfies $\left(*_{n}\right)$, then $H_{n}^{T}\left(X \bigcup_{A} Y\right) \cong H_{n}^{T}(X) \oplus H_{n}^{T}(Y)$ canonically. (In case $A$ consists of one point, the normality is not necessary.)

PRoOF. We have retractions $r_{X}: X \cup_{A} Y \rightarrow X$ and $r_{Y}: X \cup_{A} Y \rightarrow Y$ such that $r_{X}(Y)=r_{Y}(X)=A$. Let $i_{X}: X \rightarrow X \bigcup_{A} Y$ and $i_{Y}: Y \rightarrow X \bigcup_{A} Y$ be the inclusions. Let $\varphi: Z_{n}\left(X \cup_{A} Y\right) \rightarrow H_{n}^{T}(X) \oplus H_{n}^{T}(Y)$ be the homomorphism defined by :

$$
\varphi(u)=\left(r_{X \#}(u)+\overline{\left.B_{n}(X)\right)}+\left(r_{Y \#}(u)+\overline{\left.B_{n}(Y)\right)} .\right.\right.
$$

Since $r_{Y}(X) \subset A$ and $A$ is acyclic, $r_{Y *}^{T} i_{X *}^{T}=0$. On the other hand, $r_{X *}^{T}{ }^{\circ} i_{X *}^{T}=\mathrm{id}$. Then $\varphi\left(i_{X \#}(u)\right)=u+\overline{B_{n}(X)}$ for each $u \in Z_{n}(X)$. A similar statement holds for $Y$. Therefore, $\varphi$ is surjective. We shall show that $\operatorname{Ker} \varphi=\overline{B_{n}(X \cup Y)}$. Then, $\varphi$ induces the desired isomorphism. First we have

$$
\overline{B_{n}(X \cup Y)} \cap r_{X}^{-1}\left(\overline{\left.B_{n}(X)\right)} \cap r_{Y}^{-1}\left(\overline{B_{n}(Y)}\right)=\operatorname{Ker} \varphi .\right.
$$


To see $\operatorname{Ker} \varphi \subset \overline{B_{n}\left(X \bigcup_{A} Y\right)}$, let $z=\sum_{k=0}^{m} \lambda_{k} u_{k} \in Z_{n}\left(X \bigcup_{A} Y\right)\left(u_{k} \in C\left(\Delta_{n}, X \bigcup_{A} Y\right), \lambda_{k}= \pm 1\right)$ such that $\varphi(z)=0$. Since $r_{X \#}(z) \in \overline{B_{n}(X)}$ and $r_{Y \#}(z) \in \overline{B_{n}(Y)}$, it suffices to show

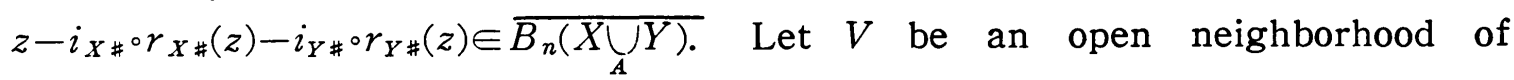
$z-i_{X \#} \circ r_{X \#}(z)-i_{Y \#} \circ r_{Y \#}(z)$. Choose an open neighborhood $U$ of $z$ so that $U-i_{X \#} \circ r_{X \#}(U)-i_{Y \#} \circ r_{Y \#}(U) \subset V$ and then open neighborhoods $U_{k}\left(\subset C\left(\Delta_{n}, X \cup_{A} Y\right)\right)$ of $u_{k}$ so that $\sum_{k=0}^{m} \lambda_{k} U_{k} \subset U$. We want to find $v_{k} \in U_{k}(0 \leqq k \leqq m)$ so that $v-i_{X \# \circ} \circ r_{X \#}(v)-i_{Y \#} \circ r_{Y \#}(v) \in B_{n}\left(X \bigcup_{A} Y\right)$, where $v=\sum_{i=0}^{m} \lambda_{k} v_{k}$. Then $V \cap B_{n}\left(X \bigcup_{A} Y\right)$ $\neq \varnothing$.

(Case $n=1$ ) We may assume $u_{k} \in Z_{1}\left(X \bigcup_{A} Y\right.$ ) for each $k$. Now fix $k$, We may also assume $U_{k}=\bigcap_{j=1}^{l} O\left(K_{j}, W_{j}\right)$. Let $\cup_{i} O_{i}=u_{k}^{-1}\left(X \cup_{A} Y \backslash A\right)$, where $O_{i}$ are open subintervals of $\Delta_{1}$ and $O_{i} \cap O_{i^{\prime}}=\varnothing$ for $i \neq i^{\prime}$. If there are only finitely

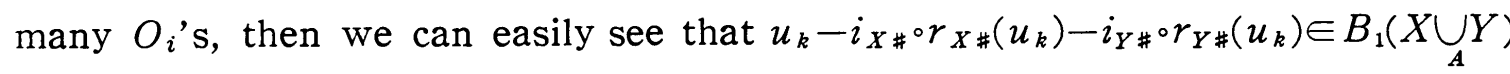
since $A$ is acyclic. In this case we let $v_{k}=u_{k}$. Otherwise we assume that the index $i$ ranges over $N$. Define $w_{n} \in C\left(\Delta_{1}, X \cup_{A} Y\right)$ by: $w_{n}\left|O_{i}=u_{k}\right| O_{i}$ for $i \leqq n$ and $w_{n}(\alpha)=r \circ u_{k}(\alpha)$ for $\alpha \notin \cup_{i=1}^{n} O_{i}$, where $r: X \bigcup_{A} Y \rightarrow A$ is the retraction defined by : $r\left|X=r_{X}\right| X$ and $r\left|Y=r_{Y}\right| Y$. We claim the existence of $w_{n} \in U_{k}$. Otherwise, there exists $0 \leqq j \leqq l$ such that $w_{n} \notin O\left(K_{j}, W_{j}\right)$ for infinitely many $w_{n}$ 's. Therefore, for infinitely many $O_{i}$ 's there exist $\alpha_{i} \in O_{i} \cap K_{j}$ such that $r \circ u_{k}\left(\alpha_{i}\right)$ $\notin W_{j}$. Let $\alpha^{*}$ be an accumulation point of $\alpha_{i}$ 's. Then $r \circ u_{k}\left(\alpha^{*}\right) \notin W_{j}, u_{k}\left(\alpha^{*}\right)$ $\in A$ and $\alpha^{*} \in K_{j}$. Hence $r \circ u_{k}\left(\alpha^{*}\right)=u_{k}\left(\alpha^{*}\right)$, which contradicts to $u_{k} \in O\left(K_{j}, W_{j}\right)$. Therefore we get $w_{n} \in U_{k}$. Now as in case there are only finitely many $O_{i}$ 's,



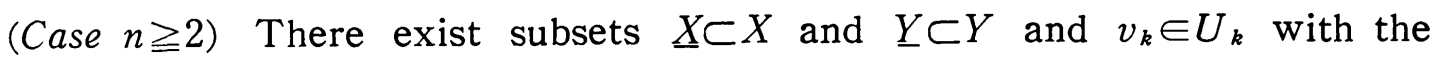
properties in $\left(*_{n}\right)$. Let $v=\sum_{k=0}^{m} \lambda_{k} v_{k}$. There is an open neighborhood $W$ of $A$ in $\underline{X}_{A} \underline{Y}$ which is deformable into $A$ in $\underline{X}_{A} \underline{Y}$. By taking barycentric subdivisions, we can take $v_{k}^{\prime} \in C\left(\Delta_{n}, X \bigcup_{A} Y\right)$ and $\lambda_{k}^{\prime}\left(0 \leqq k \leqq m^{\prime}\right)$ so that $v_{k}^{\prime}\left(\Delta_{n}\right) \subset W$, or $v_{k}^{\prime}\left(\Delta_{n}\right) \cap A=\varnothing$ and $\sum_{k=0}^{m^{\prime}} \lambda_{k}^{\prime} v_{k}^{\prime}$ is homologous to $v$ (see [3, Ch. III, §7]). By using the deformation of $W$ into $A$ and an Urysohn map with respect to $A$ and $\left(\underline{X} \bigcup_{A} \underline{Y}\right) \backslash W$, we can construct $w=\sum_{k=0}^{m^{\prime}} \lambda_{k}^{\prime} w_{k} \in Z_{n}\left(\underline{X}_{A} \underline{Y}\right)$ so that $w_{k}\left(\Delta_{n}\right) \subset \underline{X}$ or $w_{k}\left(\Delta_{n}\right) \subset \underline{Y}$ and $w$ is homologous to $v$. Then we can write $w=w_{X}+w_{Y}$, where $w_{X} \in S_{n}(\underline{X})$ and $w_{Y} \in S_{n}(\underline{Y})$. Since $\partial w_{X}=-\partial w_{Y} \in Z_{n-1}(A)$ and $A$ is acyclic, there is a $w^{*} \in S_{n}(A)$ such that $\partial w^{*}=\partial w_{X}$. Let $w^{\prime}=w_{X}-w^{*} \in Z_{n}(\underline{X})$ and $w^{\prime \prime}=w_{Y}+w^{*}$ $\in Z_{n}(\underline{Y})$. Then $w=w^{\prime}+w^{\prime \prime}$. Note that $i_{X \# \circ} \circ r_{X \#}\left(w^{\prime}\right)=w^{\prime}$ and $i_{Y \# \circ r_{Y \#}}\left(w^{\prime \prime}\right)=w^{\prime \prime}$. 
Since $A$ is acyclic, $i_{Y \# \circ} \circ r_{Y \#}\left(w^{\prime}\right), i_{X \#} \circ r_{X \#}\left(w^{\prime \prime}\right) \in B_{n}(\underline{X} \cup \underline{\underline{Y}})$. Then $i_{X \#} \circ r_{X \#}(w)-w^{\prime}$ $\in B_{n}(\underline{X} \cup \underline{Y} \underline{Y})$ and $i_{Y \#} \circ r_{Y \#}(w)-w^{\prime \prime} \in B_{n}\left(\underline{X} \bigcup_{A} \underline{Y}\right)$. Thus $w-i_{X \#} \circ r_{X \#}(w)-i_{Y \#} \circ r_{Y \#}(w)$ $\in B_{n}\left(\underline{X}_{A} \underline{Y}\right)$. Since $v$ and $w$ are homologous, $v-i_{X \# \circ} \circ r_{X \#}(v)-i_{Y \#} \circ r_{Y \#}(v) \in B_{n}\left(X \bigcup_{A} Y\right)$.

Next we are concerned with spaces obtained by attaching infinitely many spaces.

Definition 4.5. For a space $X$ with $A \subset X,(X, A)$ is primarily n-realizable, provided

$$
H_{n}^{T}(X)=\left\{u-v+\bar{B}_{n}: u, v \in C\left(\Delta_{n}, X\right), u\left(\dot{\Delta}_{n}\right), v\left(\dot{\Delta}_{n}\right) \subset A, u-v \in Z_{n}\right\} .
$$

If $X$ is $(n-1)$-connected, then $(X, A)$ is primarily $n$-realizable for any $A \subset X$ by Hurewicz's theorem. We only deal with the case that $A$ is acyclic. In this case, the condition $v\left(\dot{\Delta}_{n}\right) \subset A$ can be replaced by $v\left(\Delta_{n}\right) \subset A$. The notion "primarily $n$-realizable" is a little bit ad hoc, but it works well later on.

THEOREM 4.6. Let $X_{i}(i \in I)$ be spaces with retractions $r_{i}: X_{i} \rightarrow A_{i}$ onto copies of a contractible space $A$, or with base points $a_{i}$. Then, the following hold.

(1) If $\left(X_{i}, A\right)$ is primarily n-realizable for each $i \in I$, then both $\left(\bigvee_{i \in I}\left(X_{i}, A_{i}\right), A\right)$ and $\left(\tilde{\nabla}_{i \in I} X_{i}, a\right)$ are primary n-realizable.

(2) (Case $n=1) \quad H_{1}^{T}\left(\bigvee_{i \in I}\left(X_{i}, A_{i}\right)\right) \cong \bigoplus_{i \in I} H_{1}^{T}\left(X_{i}\right)$ canonically. Suppose that $\left(X_{i}, a_{i}\right)$ is primarily 1-realizable, then $H_{1}^{T}\left(\tilde{V}_{i \in I} X_{i}\right) \cong \tilde{\Pi}_{i \in I} H_{1}^{T}\left(X_{i}\right)$ canonically. (Case $n \geqq 2)$ If each $X_{i}$ is normal and $\left(X_{i}, A_{i}\right)$ satisfies (**), then $H_{n}^{T}\left(\bigvee_{i \in I}\left(X_{i}, A_{i}\right)\right) \cong \bigoplus_{i \in I} H_{n}^{T}\left(X_{i}\right)$. If each $\left(X_{i}, a_{i}\right)$ satisfies (**) and is primarily n-realizable, then $H_{n}^{T}\left(\tilde{V}_{i \in I} X_{i}\right) \cong \tilde{\Pi}_{i \in I} H_{n}^{T}\left(X_{i}\right)$ canonically. (In case $A=\{a\}$, the normality of spaces is not necessary.)

PRoof. First we prove the statements for $\bigvee_{i \in I}\left(X_{i}, A_{i}\right)$. Let $p_{i}: \bigvee_{i \in I}\left(X_{i}, A_{i}\right)$ $\rightarrow X_{i}(i \in I)$ be the retractions induced by the given retractions $r_{i}(i \in I)$ and $\varphi: Z_{n}\left(\bigvee_{i \in I}\left(X_{i}, A_{i}\right)\right) \rightarrow \prod_{i \in I} H_{n}^{T}\left(X_{i}\right)$ be the homomorphism defined by $: \varphi(z)(i)=$ $p_{i \#}(z)+\overline{B_{n}\left(X_{i}\right)}$. Since the image of $u \in C\left(\Delta_{n}, \bigvee_{i \in I}\left(X_{i}, A\right)\right)$ is contained in the union of finite components, we have $\operatorname{Im} \varphi \subset \bigoplus_{i \in I} H_{n}^{T}\left(X_{i}\right)$. By Theorem 44 and Lemma 4.3, we can see $H_{n}^{T}\left(\bigvee_{i \in I}\left(X_{i}, A_{i}\right)\right) \cong \bigoplus_{i \in I} H_{n}^{T}\left(X_{i}\right)$ canonically. Suppose that $\left(X_{i}, A_{i}\right)(i \in I)$ are primarily $n$-realizable. Let $c \in \bigoplus_{i \in I} H_{n}^{T}\left(X_{i}\right)$. Then, for each $i \in \operatorname{supp} c$ there exist $u_{i}, v_{i} \in C\left(\Delta_{n}, X_{i}\right)$ such that $\varphi\left(u_{i}-v_{i}\right)=c(i)$. Since $A$ is contractible, taking small simplexes in $\Delta_{n}$ of the same number as supp $c$ and using $u_{i}, v_{i}$, we can define $u, v \in C\left(\Delta_{n}, \bigvee_{i \in I}\left(X_{i}, A_{i}\right)\right)$ so that $\varphi(u-v)=c$. Therefore, $\left(\bigvee_{i \in I}\left(X_{i}, A_{i}\right), A\right)$ becomes primarily $n$-realizable. 
Next we prove the statements for $\tilde{V}_{i \in I} X_{i}$. Let $p_{i}$ and $\varphi$ as above. Then $\varphi\left(\overline{B_{n}}\right)=0$. Since the image of any $u \in C\left(\Delta_{n}, \widetilde{V}_{i \in I} X_{i}\right)$ is contained in a countable union of $X_{i}$ 's by the separability of $\Delta_{n}$, we have $\operatorname{Im} \varphi \subset \tilde{\Pi}_{i \in I} H_{n}^{T}\left(X_{i}\right)$. To see $H_{n}^{T}\left(\tilde{\vee}_{i \in I} X_{i}\right) \cong \tilde{\Pi}_{i \in I} H_{n}^{T}\left(X_{i}\right)$, if suffices to show that $\operatorname{Ker} \varphi \in \overline{B_{n}}$ and $\tilde{\Pi}_{i \in I} H_{n}^{T}\left(X_{i}\right)$ $\subset \operatorname{Im} \varphi$.

Suppose that $\varphi(z)=0$ for $z=\sum_{k=0}^{m} \lambda_{k} u_{k} \in Z_{n}\left(\lambda_{k} \in Z, u_{k} \in C\left(\Delta_{n}, \tilde{V}_{i \in I} X_{i}\right)\right)$. Then, $p_{i \#}(z) \in \overline{B_{n}\left(X_{i}\right)}$ for every $i$. For any neighborhood $U$ of $z, u_{k}(0 \leqq k \leqq m)$ have neighborhoods $U_{k}\left(\subset C\left(\Delta_{n}, \tilde{V}_{i \in I} X_{i}\right)\right)$ such that $\sum_{k=0}^{m} \lambda_{k} U_{k} \subset U$. We may assume there is a finite subset $F$ of $I$ such that $U_{k}$ 's depend on $\vee_{i \in F} X_{i}$. Let $p_{F}: \tilde{\vee}_{i \in I} X_{i} \rightarrow \bigvee_{i \in F} X_{i}$ be the retraction and $i_{F}: \bigvee_{i \in F} X_{i} \rightarrow \tilde{\vee}_{i \in I} X_{i}$ the inclusion map. Then $i_{F \#} \circ p_{F \#}\left(u_{k}\right) \in U_{k}$ and $\sum_{k=0}^{m} \lambda_{k} p_{F \#}\left(u_{k}\right) \in \overline{B_{n}\left(\bigvee_{i \in F \#} X_{i}\right.}$ by Theorem 4.4. Now $i_{F \# \circ} p_{F \#}\left(\sum_{k=0}^{m} \lambda_{k} u_{k}\right) \in \overline{B_{n}\left(\widetilde{V}_{i \in I} X_{i}\right)}$ and $i_{F \# \circ} p_{F \#}\left(\sum_{k=0}^{m} \lambda_{k} u_{k}\right)=\sum_{k=0}^{m} \lambda_{k} i_{F \#}$ $\circ \phi_{F \#}\left(u_{k}\right) \in \sum_{k=0}^{m} \lambda_{k} U_{k} \subset U$. Thus, $U \cap \overline{B_{n}} \neq \varnothing$ hence $z \in \overline{B_{n}}$.

Now, let $c \in \tilde{\Pi}_{i \in I} H_{n}^{T}\left(X_{i}\right)$. Then, $I_{0}=\operatorname{supp} c$ is countable. Since each $\left(X_{i}, a_{i}\right)$ is primarily $n$-realizable, there exist $u_{i}, v_{i} \in C\left(\Delta_{n}, X_{i}\right)\left(i \in I_{0}\right)$ such that $c(i)=$ $=u_{i}-v_{i}+\overline{B_{n}\left(X_{i}\right)}$ and $u_{i}\left(\dot{\Delta}_{n}\right)=v_{i}\left(\dot{\Delta}_{n}\right)=a_{i}$. We take small simplexes $E_{i} \subset \Delta_{n}$ ( $i \in I_{0}$ ) so that each $E_{i}$ is similar to $\Delta_{n}$ with orientation preserving similar maps $f_{i}: E_{i} \rightarrow \Delta_{n}$ and $E_{i} \cap E_{j}=\varnothing$ for $i \neq j$. Define $u, v \in C\left(\Delta_{n}, \tilde{V}_{i \in I} X_{i}\right)$ by $: u \mid E_{i}=$ $u_{i} \circ f_{i}, v \mid E_{i}=v_{i} \circ f_{i}\left(i \in I_{0}\right)$ and $u(\alpha)=v(\alpha)=a$ for $\alpha \notin \cup_{i \in I_{0}} E_{i}$. Since the diadiameters of $E_{i}$ 's converge to $0, u$ and $v$ are continuous. For each $i, p_{i \#}(u) \mid E_{i}$ $=u_{i} \circ f_{i}, p_{i \#}(v) \mid E_{i}=v_{i} \circ f_{i}$ and $p_{i \#}(u)\left(\Delta_{n} \backslash E_{i}\right)=p_{i \#}(v)\left(\Delta_{n} \backslash E_{i}\right)=a$. Since $p_{i \#}(u)$ and $p_{i \#}(v)$ are homotopic to $u_{i}$ and $v_{i}$ respectively, $p_{i \#}(u)-u_{i}, p_{i \#}(v)-v_{i} \in B_{n}\left(X_{i}\right)$. Thus we have

$$
c(i)=p_{i \#}(u)-p_{i \#}(v)+\overline{B_{n}\left(X_{i}\right)}=p_{i \#}(u-v)+\overline{B_{n}\left(X_{i}\right)}
$$

and consequently $\varphi(u-v)=c$. We have not only shown $\tilde{\Pi}_{i \in I} H_{n}^{T}\left(X_{i}\right) \subset \operatorname{Im} \varphi$ but also $\left(\tilde{\nabla}_{i \in I} X_{i}, a\right)$ is primarily $n$-realizable.

Definition 4.7. For a space $X$ with base point $a$, the $X$-piled spaces are spaces with base point inductively defined as follows:

(1) $X$ is an $X$-piled space with base point $a$;

(2) If $X_{i}(i \in I)$ are $X$-piled spaces with base points $a_{i}$, then both $\vee_{i \in I} X_{i}$ and $\bigvee_{i \in I} X_{i}$ are $X$-pilled spaces.

Along the definition of $X$-piled spaces, we define their types and orders. A rigorous reader should think that $X$-piled spaces are not just pointed spaces, but pointed spaces with their construction. However, in some cases we confuse them for short expression. Types are pairs $(\mu, \boldsymbol{P}),(\mu, \boldsymbol{S})$ and $(\mu, \boldsymbol{M})$ of ordinals 
$\mu$ and letters $\boldsymbol{P}, \boldsymbol{S}$ and $\boldsymbol{M}$. The partial order $<$ for types is defined as follows: $(\mu, \Gamma)<(\nu, \nabla)$ for $\mu<\nu$ and $\Gamma, \nabla=\boldsymbol{P}, \boldsymbol{M}$ or $\boldsymbol{S} ;(\mu, \boldsymbol{P})<(\mu, \boldsymbol{M}) ;(\mu, \boldsymbol{S})<(\mu, \boldsymbol{M})$. We identify the types $(0, \boldsymbol{P})$ and $(0, \boldsymbol{S})$ as a special case. The supremum $\sup ^{+} S$ of a set of ordinals $S$ is the least ordinal which is strictly greater than every ordinal in $S$.

Definition 4.8. For an $X$-piled spaces $Y$, the type $\operatorname{ty}(Y)$ is defined as follows and the ordinal of $\operatorname{ty}(Y)$ is called the order of $Y$ and denoted by $o(Y)$.

$\left(1^{*}\right) \quad \operatorname{ty}(X)=(0, \boldsymbol{P})=(0, \boldsymbol{S})$;

$\left(2^{*}\right)$ Let $X_{i}(i \in I)$ be $X$-piled spaces of type $\operatorname{ty}\left(X_{i}\right)$, where $|I| \geqq 2$.

Case (a): $\sup ^{*}\left\{\mathrm{o}\left(X_{i}\right): i \in I\right\}=1$.

$$
\begin{aligned}
& \operatorname{ty}\left(\bigvee_{i \in I} X_{i}\right)= \begin{cases}(1, \boldsymbol{S}) & \text { if } I \text { is infinite; } \\
(0, \boldsymbol{M}) & \text { otherwise. }\end{cases} \\
& \operatorname{ty}\left(\widetilde{\vee}_{i \in I} X_{i}\right)= \begin{cases}(1, \boldsymbol{P}) & \text { if } I \text { is infinite; } \\
(0, \boldsymbol{M}) & \text { otherwise. }\end{cases}
\end{aligned}
$$

Case (b): $\sup ^{*}\left\{\mathrm{o}\left(X_{i}\right): i \in I\right\}=\mu+1$ for $\mu \geqq 1$.

$$
\begin{aligned}
& \operatorname{ty}\left(\vee_{i \in I} X_{i}\right)= \begin{cases}(\mu+1, \boldsymbol{S}) & \text { if } I_{P} \text { is infinite; } \\
(\mu, \boldsymbol{S}) & \text { if } I_{P} \text { is empty; } \\
(\mu, \boldsymbol{M}) & \text { otherwise, }\end{cases} \\
& \operatorname{ty}\left(\tilde{\vee}_{i \in I} X_{i}\right)= \begin{cases}(\mu+1, \boldsymbol{P}) & \text { if } I_{S} \text { is infinite; } \\
(\mu, \boldsymbol{P}) & \text { if } I_{S} \text { is empty; } \\
(\mu, \boldsymbol{M}) & \text { otherwise, }\end{cases}
\end{aligned}
$$

where $I_{P}=\left\{i: \operatorname{ty}\left(X_{i}\right)=(\mu, \boldsymbol{P})\right.$ or $\left.(\mu, \boldsymbol{M})\right\}$ and $I_{S}=\left\{i: \operatorname{ty}\left(X_{i}\right)=(\mu, \boldsymbol{S})\right.$ or $\left.(\mu, \boldsymbol{M})\right\}$. Case (c): $\sup \left\{0\left(X_{i}\right): i \in I\right\}=\mu$ a limit ordinal.

$$
\operatorname{ty}\left(\bigvee_{i \in I} X_{i}\right)=(\mu, S) \text { and } \operatorname{ty}\left(\tilde{\vee}_{i \in I} X_{i}\right)=(\mu, P) .
$$

Since types of $X$-piled spaces are defined along the inductive definition, it is possible that spaces of the same homotopy type have different types. However, under some condition on $(X, a)$, types of $X$-piled spaces are quasi-homotopy invariant and so homotopy invariant. To state the condition we need a definition about groups, which is a version of a notion in $[4, \mathrm{p} .189]$. We refer the reader to [11] for undefined notions about groups. Further results we need will be proved or mentioned in the appendix.

Definition 4.9. For a group $A$, the maximal divisible subgroup of $A$ is 
denoted by $D(A)[10$, p. 100]. The properties $(\mathrm{P})$ and $(\mathrm{S})$ are defined as follows: (P) The group $n ! \cdot \Pi_{N} A / D(A)$ is not isomorphic to a summand of $n ! \cdot(A / D(A))^{m}$ for any $m, n \in \boldsymbol{N}$.

(S) The group $n ! \cdot \oplus_{N} A / D(A)$ is not isomorphic to a summand of $n ! \cdot(A / D(A))^{m}$ for any $m, n \in \boldsymbol{N}$.

If a group of finite rank is not isomorphic to a direct sum of a divisible group and a bounded group, then it satisfies both (P) and (S). Especially, a reduced torsion-free group of finite rank satisfies both.

THEOREM 4.10. Suppose that $(X, a)$ is primarily $n$-realizable. In case $n \geqq 2$, we also assume $(X, a)$ satisfies (**). Let $Y$ and $Y^{\prime}$ be $X$-piled spaces. If there exist continuous maps $f: Y \rightarrow Y^{\prime}$ and $g: Y^{\prime} \rightarrow Y$ such that $f \circ g \sim_{q} i d_{Y \cdot}$, then $\operatorname{ty}(Y) \leqq$ $\operatorname{ty}\left(Y^{\prime}\right)$ under the following conditions:

$H_{n}^{T}(X)$ satisfies $(\Gamma)$ and

(a) $\operatorname{ty}\left(Y^{\prime}\right)=(m, \nabla)$ for an odd $m \in N$;

(b) $\operatorname{ty}\left(Y^{\prime}\right)=\left(m, I^{\prime}\right)$ for an even $m \in N$; or

(c) $o\left(Y^{\prime}\right)$ is infinite,

where (1) $\boldsymbol{\Gamma}=\boldsymbol{P}$ and $\boldsymbol{\nabla}=\boldsymbol{S}$; or (2) $\Gamma=\boldsymbol{S}$ and $\boldsymbol{\nabla}=\boldsymbol{P}$.

Particularly $\operatorname{ty}(Y) \leqq \operatorname{ty}\left(Y^{\prime}\right)$ in case $H_{n}^{T}(X)$ satisfies both $(\mathrm{P})$ and $(\mathrm{S})$ and $o\left(Y^{\prime}\right)$ $\geqq 1$. Therefore, in this case the type is quasi-homotopy invariant for $X$-piled spaces of nonzero order. In addition if $H_{n}^{T}(X)$ is of finite rank, the condition $o\left(Y^{\prime}\right) \geqq 1$ is not necessary in the above.

Proof. By Theorem 3.8, $H_{n}^{T}(Y)$ is isomorphic to a summand of $H_{n}^{T}\left(Y^{\prime}\right)$. Let $A=H_{n}^{T}(X)$. By Theorem 4.6 and the definition of type for $\sigma-\operatorname{Reid}(A) \operatorname{groups}$ in the appendix, $H_{n}^{T}(Y)$ and $H_{n}^{T}\left(Y^{\prime}\right)$ belong to the $\sigma$-Reid class of $A$ and $\operatorname{ty}(Y)$ $=\operatorname{ty}\left(H_{n}^{T}(Y)\right)$ and $\operatorname{ty}\left(Y^{\prime}\right)=\operatorname{ty}\left(H_{n}^{T}\left(Y^{\prime}\right)\right)$. Therefore, the theorem follows from Theorem A.7 and Corollary A.8.

Ralph [22] has defined a factor of singular chain and homology groups $H A$ and $H M$. He showed that $H M$ can be used to detect the anomalous singular homology constructed by Barratt and Milnor [2], which is $H_{q}\left(\widetilde{V}_{N} \boldsymbol{S}^{r}\right)$. Though $H M(X)$ and $\operatorname{Ker} \sigma_{X}$, in the present paper, are different in general, $\operatorname{Ker} \sigma_{X}$ can also be used to detect such a phenomenon. To see this, we show the following.

Proposition 4.11. Let $X$ be the inverse limit $\varliminf_{(1 ;}\left(X_{i}, r_{i j}, I\right)$ of subspaces $X_{i}$ of $X$ such that each bonding map $r_{i j}: X_{j} \rightarrow X_{i}$ is a retraction. Then $H_{n}^{T}(X)$ is naturally isomorphic to a subgroup of $\varliminf_{(}\left(H_{n}^{T}\left(X_{i}\right), r_{i j *}^{T}, I\right)$. 
Proof. Let $r_{i}: X \rightarrow X_{i}(i \in I)$ be the retractions and define $\varphi: Z_{n}(X) \rightarrow$ $\varliminf\left(H_{n}^{T}\left(X_{i}\right), r_{i j *}^{T}, I\right)$ by : $r_{i *}^{T} \circ \varphi(z)=r_{i \#}(z)+\overline{B_{n}\left(X_{i}\right)}$ for $i \in I$. Let $z=\sum_{i=1}^{m} \varepsilon_{k} u_{k}$ $\left(u_{k} \in C\left(\Delta_{n}, X\right), \varepsilon_{k}= \pm 1\right)$ such that $r_{i *}^{T} \circ \varphi(z)=0$ for all $i \in I$. For any neighborhood $U$ of $z$, there exist neighborhoods $U_{k} \subset C\left(\Delta_{n}, X\right)$ of $u_{k}$ such that $\sum_{k=1}^{m} \varepsilon_{k} U_{k} \subset U$. Observe $C\left(\Delta_{n}, X\right)=\lim _{(}\left(C\left(\Delta_{n}, X_{i}\right),\left(r_{i j}\right)_{\#}, I\right)$. Then there exist $j \in I$ and neighborhood $V_{k}$ of $r_{j \#}\left(u_{k}\right)(1 \leqq k \leqq m)$ such that $r_{j \#}^{-1}\left(V_{k}\right) \subset U_{k}$. Since $r_{j}$ is a retraction, $e_{\#} \circ r_{j \#}\left(u_{k}\right) \in r_{j \#}^{-1}\left(V_{k}\right) \subset U_{k}$ where $e: X_{j} \rightarrow X$ is the inclusion, hence $e_{\#} \circ r_{j \#}(z)$

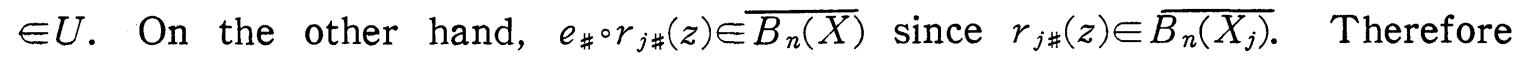
$z \in \overline{B_{n}(X)}$.

Corollary 4.12. Let $X=\lim \left(X_{i}, r_{i j}, I\right)$, where each $X_{i}$ is a subspace of $X$ and $r_{i j}: X_{j} \rightarrow X_{i}$ is a retraction. In case $H_{n}^{T}\left(X_{i}\right)=\{0\}$ for all $i \in I, H_{n}^{T}(X)=\{0\}$ and consequently $\operatorname{Ker} \sigma_{X}=H_{n}(X)$.

By this corollary $H_{q}^{T}\left(\tilde{\nabla}_{N} S^{r}\right)=\{0\}$ for $q>r$ which implies Ker $\sigma_{X}=H_{q}\left(\tilde{V}_{N} S^{r}\right)$ $\neq\{0\}$ with $q \equiv 1 \bmod (r-1), q>1, r>1 \quad[2$, Theorem 1$]$.

REMARK 4.13. In Section 3, we have shown the difference among the Čech homology group $\breve{H}_{0}(X)$, the singular homology group $H_{0}(X)$ and $H_{0}^{T}(X)$. Here we demonstrate examples which show the difference among $\check{H}_{1}(X), H_{1}(X)$ and $H_{1}^{T}(X)$.

(1) Let $D=\left\{(x, y): x^{2}+y^{2} \leqq 1\right\}$ and $\boldsymbol{S}^{1}=\dot{D}=\left\{(x, y): x^{2}+y^{2}=1\right\}$. Define $X=$ $\boldsymbol{S}^{1} \times\{0\} \cup \bigcup_{n \in N} D \times\{1 / n\}$. Then $H_{1}(X) \cong \boldsymbol{Z}$ and $\check{H}_{1}(X)=H_{1}^{T}(X)=0$.

(2) Let $X$ be the example space due to H.B. Griffiths [23, p. 59], i.e., $X=$ $Y \cup Y^{\prime}$ the subspace of $\boldsymbol{R}^{3}$ where $Y=\left\{(x, y, z): 0 \leqq z \leqq 1,(x-(1-z) / n)^{2}+y^{2}=\right.$ $\left.(1-z)^{2} / n^{2}, n \in \boldsymbol{N}\right\}$ and $Y^{\prime}$ is the reflection of $Y$ through the origin of $\boldsymbol{R}^{3}$. Then, $\pi_{1}(X) \neq 0$ but also $H_{1}(X) \neq 0$. On the other hand, $\check{H}_{1}(X)=H_{1}^{T}(X)=0$.

(3) Let $X=\widetilde{V}_{I} \boldsymbol{S}^{1}$. Then $\check{H}_{1}(X) \cong \boldsymbol{Z}^{I}$ and $H_{1}^{T}(X) \cong \tilde{\Pi}_{I} \boldsymbol{Z}$. The first author has shown that in case $I$ is infinite $H_{1}(X)$ contains $\oplus_{2 \times_{0}} \boldsymbol{Q}$ as a subgroup and hence as a summand, where $\boldsymbol{Q}$ is the group of the rational [25, Theorem 4.14 and Theorem A. 1]. It is an interesting question whether $H_{1}(X)$ is torsion-free or not.

(4) Since $H_{1}^{T}$ is a factor of $H_{1}$, one may think that $H_{1}^{T}$ gives us less information than $H_{1}$. However, it is not true. Let $\boldsymbol{H}$ be the so-called Hawaiian earing, i.e. $\boldsymbol{H}=X$ with $I=\boldsymbol{N}$ in (3), and let $x$ be a point of $\boldsymbol{H}$ such that $\boldsymbol{H}$ is locally simply connected at $x$. Then $H_{1}(\boldsymbol{H}) \cong A \oplus_{2}{\aleph_{0}}_{0} \boldsymbol{Q}$ by (3). Take a simplicial complex $Y$ with $y \in Y$ so that $H_{1}(Y) \cong \boldsymbol{Q}$. Let $Z$ be the one point union $(\boldsymbol{H}, x) \vee(Y, y)$. Then $Z$ is locally simply connected at the common point. 
Therefore, $H_{1}(Z) \cong H_{1}(\boldsymbol{H}) \oplus H_{1}(Y) \cong A \oplus\left(\oplus_{2 \times_{0}} \boldsymbol{Q}\right) \oplus \boldsymbol{Q} \cong H_{1}(\boldsymbol{H})$. On the other hand, $H_{1}^{T}(Z) \cong H_{1}^{T}(\boldsymbol{H}) \oplus H_{1}(Y) \cong Z^{N} \oplus \boldsymbol{Q}$, which is apparently not isomorphic to $H_{1}^{T}(\boldsymbol{H})$ $\cong Z^{N}$.

\section{Realizing the groups $C(X, \boldsymbol{Z})$}

In this section, we show the following.

THEOREMi 5.i. Let $X$ be a bounded subset of the real line $\boldsymbol{R}$. Then $H_{n}^{T}\left(\boldsymbol{R}^{n+1} \backslash X \times\{0\}\right) \cong C(X, \boldsymbol{Z})$ canonically, where $0=(0, \cdots, 0) \in \boldsymbol{R}^{n}$.

Proof. We indentify $Y \subset \boldsymbol{R}$ with $Y \times\{0\} \subset \boldsymbol{R}^{n+1}$. For each $x \in X$, let $i_{x}: \boldsymbol{R}^{n+1} \backslash X \rightarrow \boldsymbol{R}^{n+1} \backslash\{x\}$ be the inclusion. Identifying $\boldsymbol{Z}$ with $H_{n}^{T}\left(\boldsymbol{R}^{n+1} \backslash\{x\}\right)$ canonically, we define a homomorphism $\varphi: Z_{n}\left(\boldsymbol{R}^{n+1} \backslash X\right) \rightarrow \boldsymbol{Z}^{X}$ by $\varphi(z)(x)=$ $\left(i_{x}\right)_{*}^{T}\left(z+\overline{B_{n}}\right)$. Let $z=\sum_{k=0}^{m} \lambda_{k} u_{k} \in Z_{n}$, where $\lambda_{k} \in Z$ and $u_{k} \in C\left(\Delta_{n}, \boldsymbol{R}^{n+1} \backslash X\right)$. Each $x \in X$ has a contractible neighborhood $U$ in $\boldsymbol{R}^{n+1}$ such that $U \cap \operatorname{Im} u_{k}=\varnothing$ for every $k$. Therefore, $\left(i_{x}\right)_{*}^{T}\left(z+\overline{B_{n}}\right)$ is constant in $U$ as a function of $x$, which implies $\operatorname{Im} \varphi \subset C(X, Z)$. And clearly $\overline{B_{n}} \subset \operatorname{Ker} \varphi$. Then, it suffices to show that (i) $\operatorname{Ker} \varphi \subset \overline{B_{n}}$ and (ii) $C(X, Z) \subset \operatorname{Im} \varphi$.

(i) Let $z \in \operatorname{Ker} \varphi$ be written as above. For any open neighborhood $V$ of $z$, there exists $\varepsilon>0$ such that $\rho\left(u_{k}, u_{k}^{\prime}\right)<\varepsilon(0 \leqq k \leqq m)$ implies $\sum_{k=0}^{m} \lambda_{k} u_{k}^{\prime} \in V$, where $\rho$ is the sup-metric induced from Euclidean metric. Choose $x_{0}<x_{1}<\cdots<x_{M}$ in $\boldsymbol{R}$ so that $X \subset\left(x_{0}, x_{M}\right)$ and $x_{i}-x_{i-1}<\varepsilon / n+1 \quad(1 \leqq i \leqq M)$ and $x_{i} \in X$ implies $\left(x_{i}-\delta, x_{i}+\delta\right) \subset X$ for some $\delta>0$ and $x_{i}, x_{i+1} \in X$ implies $\left[x_{i}, x_{i+1}\right] \subset X$. Let $K=$ $\left\{i:\left(x_{i}, x_{i+1}\right) \cap X \neq \varnothing\right\}$. For each $i \in K$, let $D_{i}=\left(x_{i}, x_{i+1}\right) \times(-\varepsilon / n+1, \varepsilon / n+1)^{n}$ and take $y_{i} \in\left(x_{i}, x_{i+1}\right) \cap X$ so that $x_{i} \in X$ implies $\left[x_{i}, y_{i}\right] \subset X$ and $x_{i+1} \in X$ implies $\left[y_{i}, x_{i+1}\right] \subset X$. Let

$$
h: \boldsymbol{R}^{n+1} \backslash\left\{y_{i}: i \in K\right\} \longrightarrow \boldsymbol{R}^{n+1} \bigcup_{i \in K} D_{i}
$$

be the radial deformation retraction, i.e. for each $\boldsymbol{a}$ on the boundary of $D_{i}, h$ maps the segment between $y_{i}$ and $\boldsymbol{a}$ to the point $\boldsymbol{a}$. According to the choice of $x_{i}$ and $y_{i}$, we have

$$
h\left(\boldsymbol{R}^{n+1} \backslash X\right)=\boldsymbol{R}^{n+1} \backslash\left(X \cup \bigcup_{i \in K} D_{i}\right) \quad\left(\subset \boldsymbol{R}^{n+1} \backslash X\right) .
$$

which has a homotopy type of an $n$-dimensional bouquet. Since $\left(i_{y_{i}}\right)_{*}^{T}\left(h_{\#}(z)\right)=$ $\left(i_{y_{i}}\right)_{*}^{T}(z)=0$ for each $i \in E$, we can see $h_{\#}(z) \in B_{n}\left(h\left(\boldsymbol{R}^{n+1} \backslash X\right)\right) \subset B_{n}\left(\boldsymbol{R}^{n+1} \backslash X\right)$. Since $h$ moves points in distance less than $\varepsilon, \rho\left(u_{k}, h_{\#}\left(u_{k}\right)\right)<\varepsilon$ for each $k$. Then $h_{\#}(z)=\sum_{k=0}^{m} \lambda_{k} h_{\#}\left(u_{k}\right) \in V$. Thus we have $\operatorname{Ker} \varphi \subset \overline{B_{n}}$.

(ii) To see $C(X, Z) \subset \operatorname{Im} \varphi$, let $f \in C(X, Z)$. There exist pairwise disjoint 
open intervals $I_{k}(k \in N)$ such that $X \subset \cup_{k \in N} I_{k}$ and $f$ is constant on each $X \cap I_{k}$. Let $D_{k}$ be the $(n+1)$-ball with center $x_{k}$ and its diametrical axis $I_{k}$, and $C_{k}$ the boundary $n$-sphere of $D_{k}$. Take an $n$-simplex $E \subset \boldsymbol{R}^{n} \times\{0\}$ large enough so that $E$ contains all $C_{k} \cap \boldsymbol{R}^{n} \times\{0\}$ in its interior. Take a homeomorphism $g: \Delta_{n} \rightarrow E$ and for each $k$ let $u_{k}, v_{k}: \Delta_{n} \rightarrow E \cup D_{k} \backslash\left\{x_{k}\right\}$ be continuous maps such that:

(1) $u_{k}(\alpha)=v_{k}(\alpha)=g(\alpha)$ for $\alpha \notin g^{-1}\left(D_{k} \cap \boldsymbol{R}^{n} \times\{0\}\right)$;

(2) $u_{k}$ maps $g^{-1}\left(D_{k} \cap \boldsymbol{R}^{n} \times\{0\}\right)$ to $C_{k}$;

(3) $v_{k}$ maps $g^{-1}\left(D_{k} \cap \boldsymbol{R}^{n} \times\{0\}\right)$ to $C_{k} \cap \boldsymbol{R}^{n} \times\{y: y \leqq 0\}$ homeomorphically ;

(4) $\quad\left(i_{x_{k}}\right)_{*}^{T}\left(u_{k}-v_{k}+\overline{B_{n}}\right)=f(k) \in H_{n}^{T}\left(\boldsymbol{R}^{n+1} \backslash\left\{x_{k}\right\}\right)$.

Finally, let $u, v: \Delta_{n} \rightarrow \boldsymbol{R}^{n+1} \backslash X$ be the maps defined by $u(\alpha)=v(\alpha)=i(\alpha)$ for $\alpha \notin \bigcup_{k \in N} g^{-1}\left(D_{k} \cap \boldsymbol{R}^{n} \times\{0\}\right)$; and $u(\alpha)=u_{k}(\alpha)$ and $v(\alpha)=v_{k}(\alpha)$ for $\alpha \in g^{-1}\left(D_{k} \cap \boldsymbol{R}^{n}\right.$ $\times\{0\})$ and each $k \in N$. Then, $u-v \in Z_{n}$ and $\varphi(u-v)=f$.

REMARK 5.2. In case $X$ is unbounded in the theorem, we get $H_{n}^{T}\left(\boldsymbol{R}^{n+1} \backslash X\right)$ $\cong(f \in C(X, \boldsymbol{Z}): \operatorname{supp} f$ is bounded $\}$, where supp $f=\{x \in X: f(x) \neq 0\}$. Let $\boldsymbol{L}^{+}$be Cantor's long ray $\left[21\right.$, p. 643] and $X \subset L^{+}$. Similarly we get an isomorphism $H_{n}^{T}\left(\boldsymbol{L}^{+} \times \boldsymbol{R} \backslash X \times\{0\}\right) \cong\{f \in C(X, \boldsymbol{Z})$ : supp $f$ is bounded $\}$. Thus, the group in [9, Theorem 4.6] is naturally realized by $H_{1}^{T}(Y)$.

It will be shown in Proposition A.11 that $C(X, Z)$ is a $\sigma-\operatorname{Reid}(\boldsymbol{Z}) \operatorname{group}$ for any scattered subspace $X$ of $\boldsymbol{R}$. On the other hand neither $C(\boldsymbol{Q}, \boldsymbol{Z})$ nor $C(\boldsymbol{R} \backslash \boldsymbol{Q}, \boldsymbol{Z})$ belongs to the $\sigma$-Reid class of $\boldsymbol{Z}$ by the same reason as in [5]. Hence, neither $\boldsymbol{R}^{2} \backslash \boldsymbol{Q}$ nor $\boldsymbol{R}^{2} \backslash(\boldsymbol{R} \backslash \boldsymbol{Q})$ is quasi-homotopic to $\boldsymbol{R}^{2} \backslash X$ for any scattered subspace $X$ of $\boldsymbol{R}$ by Theorem 5.1 .

\section{Spatial homomorphisms}

For spaces $X$ and $Y$, a homomorphism $h: H_{n}^{T}(X) \rightarrow H_{n}^{T}(Y)\left(h: H_{n}(X) \rightarrow H_{n}(Y)\right)$ is called spatial if there exists a continuous map $f: X \rightarrow Y$ such that $h=f_{*}^{T}$ $\left(h=f_{*}\right)$. In this section, we show that any homomorphism from $H_{n}^{T}(X)$ to $H_{n}^{T}(Y)$ is spatial for any $\boldsymbol{S}^{n}$-piled spaces $X$ and $Y$. As we shall show later, this does not hold for singular homology groups. Recall that an $\boldsymbol{S}^{n}$-piled space $X$ consists of copies of $\boldsymbol{S}^{n}$ with identified base points. By $\boldsymbol{e}$, we denote the base point of $\boldsymbol{S}^{n}$ and also of $X$. We call each copy of $\boldsymbol{S}^{n}$ a basic component of $X$. Our result of this section is concerned with slenderness of groups. A group $A$ is slender if for any homomorphism $h: Z^{N} \rightarrow A$ there exists $n \in N$ such that $h\left(e_{m}\right)=0$ for $m \geqq n$, where $e_{m}(m)=1$ and $e_{m}(i)=0$ for $i \neq m$ [11, XIII 94]. As is well known, it is also equivalent that for any homomorphism $h: Z^{N} \rightarrow A$ there exists 
$n \in \boldsymbol{N}$ such that $h\left(\boldsymbol{Z}^{\boldsymbol{N} \backslash n}\right)=\{0\}$.

By a straightforward induction on the definition of piled spaces, we get the following

Lemma 6.1. Let $X$ be an $\boldsymbol{S}^{n}$-piled space and $X_{\lambda}(\lambda \in \Lambda)$ be all the basic components of $X$. If $\sigma_{\lambda}: X_{\lambda} \rightarrow X_{\lambda}(\lambda \in \Lambda)$ are base point preserving continuous maps, then the induced map $\sigma: X \rightarrow X$ (i.e., $\sigma \mid X_{\lambda}=\sigma_{\lambda}$ for each $\lambda$ ) is continuous.

THEOREM 6.2. Let $X$ and $Y$ be $\boldsymbol{S}^{n}$-piled spaces. Then any homomorphism from $H_{n}^{T}(X)$ to $H_{n}^{T}(Y)$ is spatial.

Proof. In case $X=Y=\boldsymbol{S}^{n}$, the theorem holds, since any endomorphism on $H_{n}^{T}\left(\boldsymbol{S}^{n}\right)(\cong \boldsymbol{Z})$ is spatial. Our proof goes by induction on the definitions of $X$ and $Y$. In the sequel, $q_{j}: Y \rightarrow Y_{j}(j \in J)$ denote the projections in both cases $Y=\tilde{\vee}_{j \in J} Y_{j}$ and $Y=\vee_{j \in J} Y_{j}$. Let $X=\vee_{i \in I} X_{i}$ or $X=\tilde{\vee}_{i \in I} X_{i}$. Our induction hypothesis is that any homomorphism from $H_{n}^{T}(X)$ to $H_{n}^{T}\left(Y_{j}\right)$ and one from $H_{n}^{T}\left(X_{i}\right)$ to $H_{n}^{T}(Y)$ are induced by base point preserving continuous maps. We remark the following property $(+)$ of $S^{n}$ :

(+) There exist pairwise disjoint open sets $O_{m}(m \in \boldsymbol{N})$ in $\boldsymbol{S}^{n}$ and continuous maps $\boldsymbol{\sigma}_{m}: \boldsymbol{S}^{n} \rightarrow \boldsymbol{S}^{n}(m \in \boldsymbol{N})$ such that $\boldsymbol{e} \notin O_{m}, \boldsymbol{\sigma}_{m}\left(\boldsymbol{S}^{n} \backslash O_{m}\right)=\{\boldsymbol{e}\}$ and $\sigma_{m} \underset{\boldsymbol{k}}{\mathrm{id}}$.

(Case 1) $X=\boldsymbol{S}^{n}$. Let $Y=\bigvee_{j \in J} Y_{j}$. Since $H_{n}^{T}(Y) \cong \bigoplus_{j \in J} H_{n}^{T}\left(Y_{j}\right)$ canonically by Theorem 4.6 and $H_{n}^{T}\left(\boldsymbol{S}^{n}\right) \cong Z$, there exists a finite subset $F$ of $J$ such that $\operatorname{Im} h$ $\subset \bigoplus_{j \in F} H_{n}^{T}\left(Y_{j}\right)$. The induction hypothesis implies the existence of base point preserving continuous maps $f_{j}: X \rightarrow Y_{j}(j \in F)$ such that $\left(f_{j}\right)_{*}^{T}=\left(q_{j}\right)_{*}^{T} \circ h$. Take $O_{j}$ and $\sigma_{j}: X \rightarrow X$ indexed by $j \in J$ with the properties assured by $(+)$. Define $f: X \rightarrow Y$ by :

$$
f(s)= \begin{cases}f_{j} \circ \sigma_{j}(s) & \text { for } s \in O_{j}(j \in F) \\ \boldsymbol{e} & \text { otherwise. }\end{cases}
$$

Then $f$ is continuous, $\operatorname{Im} f_{*}^{T} \subset \bigoplus_{j \in F} H_{n}^{T}\left(Y_{j}\right)$ and $\left(q_{j}\right)_{*}^{T} \circ f_{*}=\left(q_{j} \circ f\right)_{*}^{T}=\left(q_{j} \circ f \circ \sigma_{j}\right)_{*}^{T}=$ $\left(f_{j}\right)_{*}^{T}=\left(q_{j}\right)_{*}^{T} \circ h$ for each $j \in F$. Hence $f_{*}^{T}=h$.

Next, let $Y=\widetilde{V}_{j \in J} Y_{j}$. Since $H_{n}^{T}(Y) \cong \tilde{\Pi}_{j \in J} H_{n}^{T}\left(Y_{j}\right)$ canonically by Theorem 4.6, there exists a countable subset $C$ of $J$ such that $\operatorname{Im} h \subset \Pi_{j \in C} H_{n}^{T}\left(y_{j}\right)$. As in the case of $\vee_{j \in J} Y_{j}$, take $f_{j}, O_{j}$ and $\sigma_{j}(j \in C)$ and define $f: X \rightarrow Y$ by:

$$
f(s)= \begin{cases}f_{j} \circ \sigma_{j}(s) & \text { for } s \in O_{j}(j \in C) \\ \boldsymbol{e} & \text { otherwise }\end{cases}
$$


Since $q_{j} \circ f$ is continuous for all $j \in J, f$ is continuous and we can conclude $f_{*}^{T}$ $=h$ as above.

(Case 2) $X=\bigvee_{i \in I} X_{i}$. By induction hypothesis, there exist base point preserving continuous maps $f_{i}: X_{i} \rightarrow Y(i \in I)$ such that $h \mid H_{n}^{T}\left(X_{i}\right)=\left(f_{i}\right)_{*}^{T}$. Define a continuous map $f: X \rightarrow Y$ by $: f \mid X_{i}=f_{i}$ for $i \in I$. Then it is easy to see that $h=f_{*}^{T}$.

(Case 3) $X=\tilde{\nabla}_{i \in I} X_{i}$. When $Y=\boldsymbol{S}^{n}, H_{n}^{T}(Y)(\cong \boldsymbol{Z})$ is a slender group. Applying Theorem 1(1) of [8] in a similar way as in the proof of Lemma A.2, we get a finite subset $E$ of $I$ and $\bar{h}: \bigoplus_{i \in E} H_{n}^{T}\left(X_{i}\right) \rightarrow H_{n}^{T}(Y)$ such that $h=\bar{h} \circ \pi_{E}$, where $\pi_{E}: \tilde{\Pi}_{i \in I} H_{n}^{T}\left(X_{i}\right) \rightarrow \bigoplus_{i \in E} H_{n}^{T}\left(X_{i}\right)$ is the projection. We get the desired continuous map $f$ through the projection as in Case 2.

Let $Y=\vee_{j \in J} Y_{j}$. By Lemma A.2 and the torsion-freeness of $H_{n}^{T}(Y)$, there exist finite subsets $E$ of $I$ and $F$ of $J$ such that $h\left(\tilde{\Pi}_{i \in I \backslash E} H_{n}^{T}\left(X_{j}\right)\right) \subset \bigoplus_{j \in F} H_{n}^{T}\left(Y_{j}\right)$. Let $X_{i \lambda}\left(\lambda \in \Lambda_{i}\right)$ be all the basic components of $X_{i}$ for each $i$. Then, $H_{n}^{T}\left(X_{i \lambda}\right)$. $\left(\lambda \in \Lambda_{i}, i \in I\right)$ correspond to all the basic components of $H_{n}^{T}(X)$. (See Definition A.9.) Since $\tilde{\nabla}_{i \in I \backslash E} X_{i}$ is a retract of $X$, by induction hypothesis there exists a base point preserving continuous map $g_{j}: \widetilde{V}_{i \in I \backslash E} X_{i} \in Y_{j}$ for each $j$ such that $\left(g_{j}\right)_{*}^{T}=\left(q_{j}\right)_{*}^{T} \circ h \mid \tilde{\Pi}_{i \in I \backslash E} H_{n}^{T}\left(X_{i}\right)$. We get $O_{i \lambda j} \subset X_{i \lambda}(j \in F)$ and $\sigma_{i \lambda j}: X_{i \lambda} \rightarrow X_{i \lambda}$ with the properties assured by $(+)$. Then there exists a continuous map $\sigma_{j}: \tilde{\nabla}_{i \in I \backslash E} X_{i}$ $\rightarrow \tilde{V}_{i \in I \backslash E} X_{i}$ induced by $\sigma_{i \lambda j}\left(i \in I \backslash E, \lambda \in \Lambda_{i}\right)$ for each $j \in F$ by Lemma 6.1. Define $f_{E}: \tilde{\nabla}_{i \in I \backslash E} X_{i} \rightarrow \bigvee_{j \in F} Y_{j}\left(\subset \bigvee_{j \in J} Y_{j}\right)$ by :

$$
f_{E}(s)= \begin{cases}g_{j^{\circ}} \sigma_{i \lambda j}(s) & \text { for } s \in O_{i \lambda j}\left(i \in I \backslash E, \lambda \in \Lambda_{i}, j \in F\right) ; \\ \boldsymbol{e} & \text { otherwise. }\end{cases}
$$

Then the continuity of $f_{E}$ follows from the continuity of $\sigma_{j}(j \in F)$. We have $h \mid \tilde{\Pi}_{i \in I \backslash E} H_{n}^{T}\left(X_{i}\right)=\left(f_{E}\right)_{*}^{T}$ by the definition and Lemma A.10, since $\left(q_{j}\right)_{*}^{T} \circ h \mid H_{n}^{T}\left(X_{i \lambda}\right)$ $=\left(g_{j}\right)_{*}^{T} \mid H_{n}^{T}\left(X_{i \lambda}\right)=\left(q_{j}\right)_{*}^{T} \circ\left(f_{E}\right)_{*}^{T}$ for $j \in J$. For each $i \in E$, there is a base point preserving continuous map $f_{i}: X_{i} \rightarrow Y$ such that $h \mid H_{n}^{T}\left(X_{i}\right)=\left(f_{i}\right)_{*}^{T}$ by induction hypothesis. Combining $f_{E}$ and $f_{i}(i \in E)$, we get the desired map.

Let $Y=\tilde{\nabla}_{j \in J} Y_{j}$. Then there exist base point preserving continuous maps $g_{j}: X \rightarrow Y_{j}(j \in J)$ such that $g_{j}(x)=y_{j}$ and $\left(q_{j}\right)_{*}^{T} \circ h=\left(g_{j}\right)_{*}^{T} . \quad$ As before for each $i \in I$ and $\lambda \in \Lambda_{i}$, there exists a countable subset $C_{i \lambda}$ of $J$ such that $h\left(H_{n}^{T}\left(X_{i \lambda}\right)\right) \subset$ $\Pi_{j \in C_{i \lambda}} H_{n}^{T}\left(Y_{j}\right)$. We get $O_{i \lambda j} \subset X_{i \lambda}\left(j \in C_{i \lambda}\right)$ and $\sigma_{i \lambda j}: X_{i \lambda} \rightarrow X_{i \lambda}$ as before. Define $f: X \rightarrow Y$ by :

$$
f(s)= \begin{cases}g_{j} \circ \sigma_{i \lambda j}(s) & \text { for } s \in O_{i \lambda j}\left(i \in I, \lambda \in \Lambda_{i}, j \in C_{i \lambda}\right) ; \\ \boldsymbol{e} & \text { otherwise. }\end{cases}
$$


For each $j$, define a continuous map $\varphi_{j}: X \rightarrow X$ by:

$$
\varphi_{j} \mid X_{i \lambda}=\sigma_{i \lambda j} \quad \text { if } j \in C_{i \lambda} ; \varphi_{j}\left(X_{i \lambda}\right)=\{x\} \text { otherwise. }
$$

Then $q_{j} \circ f=g_{j} \circ \varphi_{j}$ for each $j \in J$, hence $f$ is continuous. It follows that

$$
\begin{gathered}
\left(q_{j}\right)_{*}^{T} \circ f_{*}^{T}\left|H_{n}^{T}\left(X_{i \lambda}\right)=\left(g_{j} \circ \varphi_{j}\right)_{*}^{T}\right| H_{n}^{T}\left(X_{i \lambda}\right)=\left(g_{j}\right)_{ \pm}^{T}\left|H_{*}^{n}\left(X_{i \lambda}\right)=\left(q_{j}\right)_{*}^{T} \circ h\right| H_{n}^{T}\left(X_{i \lambda}\right) \\
\text { for } i \in I, \lambda \in \Lambda_{i} \text { and } j \in C_{i \lambda} \quad \text { and } \\
\left(q_{j}\right)_{*}^{T} \circ f_{*}^{T}\left|H_{n}^{T}\left(X_{i \lambda}\right)=0=\left(q_{j}\right)_{*}^{T} \circ\right| H_{n}^{T}\left(X_{i \lambda}\right) \quad \text { otherwise. }
\end{gathered}
$$

Therefore $h=f_{*}^{T}$ by Lemma A.10.

Next we show that slenderness of groups can be characterized using the notion of spatial homomorphisms. Let $\boldsymbol{H}=\widetilde{V}_{i \in N} \boldsymbol{S}_{i}^{\mathbf{i}}$ be the Hawaiian earring, where $\boldsymbol{S}_{i}^{1}$ is a copy of $\boldsymbol{S}^{1}$ for $i \in \boldsymbol{N}$, and $\boldsymbol{K}(A, 1)$ be an Eilenberg-Maclane complex [23].

THEOREM 6.3. The following statements are equivalent for a group $A$.

(1) $A$ is slender;

(2) For a path connected space $X$ with $H_{1}(X) \cong A$, any homomorphism $h: H_{1}(\boldsymbol{H}) \rightarrow H_{1}(X)$ is spatial;

$\left(2^{T}\right)$ For a path connected space $X$ with $H_{1}^{T}(X) \cong A$, any homomorphism $h: H_{1}^{T}(\boldsymbol{H}) \rightarrow H_{1}^{T}(X)$ is spatial;

(3) Any homomorphism $h: H_{1}(\boldsymbol{H}) \rightarrow H_{1}(\boldsymbol{K}(A, 1))$ is spatial;

$\left(3^{T}\right)$ Any homomorphism $h: H_{1}^{T}(\boldsymbol{H}) \rightarrow H_{1}^{T}(\boldsymbol{K}(A, 1))$ is spatial.

Proof. The implications $(2) \rightarrow(3)$ and $\left(2^{T}\right) \rightarrow\left(3^{T}\right)$ are obvious. Since $H_{1}(\boldsymbol{K}(A, 1))=H_{1}^{T}(\boldsymbol{K}(A, 1))$ by Corollary 2.2, (3) $\rightarrow\left(3^{T}\right)$ follows from Proposition 1.1.

$(1) \rightarrow\left(2^{T}\right):$ By Theorem 4.6, $H_{1}^{T}(\boldsymbol{H}) \cong \Pi_{i \in N} H_{1}^{T}\left(\boldsymbol{S}_{i}^{1}\right)\left(\cong Z^{N}\right)$ naturally. There exists $n \in N$ such that $h\left(\Pi_{i \geq n} H_{1}^{T}\left(\boldsymbol{S}_{i}^{1}\right)\right)=\{0\}$. Fix a point $x \in X$. Since $X$ is path connected, there exist continuous maps $f_{i}: \boldsymbol{S}_{i}^{1} \rightarrow X(i<n)$ such that $f_{i}(\boldsymbol{e})=x$ and $\left(f_{i}\right)_{*}^{T}=h \mid H_{1}^{T}\left(\boldsymbol{S}_{i}^{1}\right)$. Define $f: \boldsymbol{H} \rightarrow X$ by $: f \mid \boldsymbol{S}_{i}^{1}=f_{i}(i<n)$ and $f\left(\bigvee_{i \geq n} \boldsymbol{S}_{i}^{1}\right)=\{x\}$. Then $h=f_{*}^{T}$.

$(1) \rightarrow(2)$ : Here we use some results and notation in [25]. By Griffith' theorem [25, Theorem A.1], $\pi_{1}(\boldsymbol{H}, \boldsymbol{e}) \cong \mathbb{X}_{N} \boldsymbol{Z}$ hence $H_{1}(\boldsymbol{H}) \cong A b\left(\mathbb{X}_{N} \boldsymbol{Z}\right)$. Since $H_{1}^{T}(\boldsymbol{H}) \cong \boldsymbol{Z}^{\boldsymbol{N}}$ canonically, $\operatorname{Ker} \sigma_{\boldsymbol{H}} \cong C_{\boldsymbol{N}} /\left(\mathbb{X}_{N} \boldsymbol{Z}\right)^{\prime}$, which is complete $\bmod -U$ by Theorems 3.3 and 4.7 of [25]. Consequently $h\left(\operatorname{Ker} \sigma_{H}\right)=\{0\}$, hence, there exists a homomorphism $\bar{h}: H_{1}^{T}(\boldsymbol{H}) \rightarrow H_{1}(X)$ such that $h=\bar{h}_{\circ} \sigma_{\boldsymbol{H}}$. Similarly as above we define $f_{i}$ 's so that $\left(f_{i}\right)_{*}=h \mid H_{1}\left(\boldsymbol{S}_{i}^{1}\right)$ and also $f$. Since $h$ only depends on the direct summands $H_{1}\left(\boldsymbol{S}_{i}^{1}\right)(1 \leqq i<n)$ of $H_{1}(\boldsymbol{H})$, we get $h=f_{*}$. 
$\left(3^{T}\right) \rightarrow(1)$ : Let $h: \boldsymbol{Z}^{N} \rightarrow A$ be a homomorphism, where we identify $\boldsymbol{Z}^{\boldsymbol{N}}$ and $A$ with $H_{1}^{T}(\boldsymbol{H})$ and $H_{1}^{T}(\boldsymbol{K}(A, 1))$ respectively. Let $f: \boldsymbol{H} \rightarrow \boldsymbol{K}(A, 1)$ be a continuous map with $f_{*}^{T}=h$. Since $\boldsymbol{K}(A, 1)$ is locally contractible, there exists $n \in N$ such that $f_{*}^{T}\left(H_{1}^{T}\left(\boldsymbol{S}_{i}^{1}\right)\right)=\{0\}$ for $i<n$. Hence $A$ is slender.

REMARK 6.4. (1) Here we show that Theorem 6.2 does not hold for singular homology groups. By [25, Theorem 4.14], $H_{1}(\boldsymbol{H})$ contains a subgroup isomorphic to $\boldsymbol{Q}$. Since $H_{n}^{T}(\boldsymbol{H}) \cong \boldsymbol{Z}^{N}$ is a torsion-free abelian group of cardi nality $2^{\times_{0}}$ and $\sigma_{H}: H_{1}(\boldsymbol{H}) \rightarrow H_{1}^{T}(\boldsymbol{H})$ is an epimorphism, there exist $2^{2 \times_{0}}$-many endomorphisms on $H_{1}(\boldsymbol{H})$. On the other hand, there exist only $2^{\times_{0}-m a n y}$ continuous maps from $\boldsymbol{H}$ to itself. Hence, not all endomorphisms are induced by continuous maps.

(2) In case $X=\tilde{\Pi}_{i \in I} \boldsymbol{S}_{i}^{1}$, any endomorphism $h$ of $H_{1}(X)\left(=H_{1}^{T}(X)\right)$ is spatial. We show this as follows. Since $\pi_{1}(X) \cong \tilde{\Pi}_{I} \boldsymbol{Z}, H_{1}(X) \cong \tilde{\Pi}_{I} \boldsymbol{Z}$. Let $p_{i}: X \rightarrow \boldsymbol{S}_{i}^{1}$ $(i \in I)$ and $p_{F}: X \rightarrow \prod_{i \in F} S_{i}^{1}(F \subset I)$ be the projections, where we consider $\boldsymbol{S}_{i}^{1}$ and $\Pi_{i \in F} \boldsymbol{S}_{i}^{1}$ as subspaces of $X$. $\operatorname{Hom}\left(\tilde{\Pi}_{i \in I} A_{i}, \boldsymbol{Z}\right) \cong \bigoplus_{i \in I} \operatorname{Hom}\left(A_{i}, \boldsymbol{Z}\right)$ [8], hence for any $i \in I$ there exists a finite subset $F_{i}$ of $I$ such that $\left(p_{i}\right)_{*} \circ h=\left(p_{i}\right)_{*} \circ \circ\left(p_{F_{i}}\right)_{*}$. Since any homomorphism from $H_{1}\left(\mathbf{I I}_{j \in F_{i}} \boldsymbol{S}_{j}^{1}\right)$ to $H_{1}\left(\boldsymbol{S}_{i}^{1}\right)$ is spatial, there exists a continuous map $f_{i}: \prod_{j \in I} \boldsymbol{S}_{j}^{1} \in \boldsymbol{S}_{i}$ such that $\left(p_{i}\right)_{*} \circ h=\left(f_{i}\right)_{*}\left(=\left(f_{i}\right)_{*}^{T}\right)$ for each $i$. Define a continuous map $f: X \rightarrow X$ by $: p_{i} \circ f=f_{i}$ for every $i$. Then $h=f_{*}$.

In case $X=\mathrm{II}_{I} S^{1}$, the situation is a little different. (Recall that this is a canonical compact abelian group.) If the cardinality of $I$ is less than the least measurable cardinal, then every endomorphism of $H_{1}(X)$ is spatial, since $\operatorname{Hom}\left(\Pi_{i \in I} A_{i}, \boldsymbol{Z}\right) \cong \bigoplus_{i \in I} \operatorname{Hom}\left(A_{i}, \boldsymbol{Z}\right) \quad[11, \S 94]$. Otherwise, there exist nonspatial endomorphisms of $H_{1}(X)\left(\cong H_{1}^{T}(X)\right)$. To see this, let $p_{i}$ and $p_{F}$ be the projections as above. Take a non-principal countably complete ultrafilter $\mathscr{I}$ on $I$ and define a homomorphism $h: H_{1}(X) \rightarrow H_{1}\left(S_{i_{0}}^{1}\right)\left(\subset H_{1}(X)\right)$ by :

$$
h\left(u+B_{1}\right)=a \quad \text { iff }\left\{i \in I: p_{i}^{\circ} u+B_{1}=a\right\} \in \mathscr{F},
$$

where $u \in C\left(\Delta_{1}, X\right) \cap Z_{1}(X)$ and $a \in H_{1}\left(\boldsymbol{S}_{i_{0}}^{1}\right)(\cong \boldsymbol{Z})$. Suppose that $h=f_{*}$ for some continuous map $f: X \rightarrow X$. Then $\left(p_{i_{0}} \circ f\right)_{*}=f_{*}$ holds. We define $u \in C\left(\Delta_{1}, \boldsymbol{S}^{1}\right) \cap$ $Z_{1}\left(\boldsymbol{S}^{1}\right)$ by $: u(x, y)(i)=(\cos 2 \pi x, \sin 2 \pi x)$ for $(x, y) \in \Delta_{1}$ and $i \in I$. Then $h\left(u+B_{1}\right)$ $\neq 0$. Let $\varepsilon>0$ be so small that $\rho(v, w)<\varepsilon$ implies that $v \widetilde{h} w$ for $v, w \in C\left(\Delta_{1}, \boldsymbol{S}^{1}\right)$ $\cap Z_{1}\left(S^{1}\right)$. Choose basic open sets $V_{1}, \cdots, V_{m} \subset X$ so that $\operatorname{Im} u \subset \cup_{j=1}^{m} V_{j}$ and $\sup \left\{\rho\left(p_{i_{0}} \circ f(x), p_{i_{0}} \circ f(y)\right): x, y \in V_{j}\right\}<\varepsilon$ for $1 \leqq j \leqq m$. Then there exists a finite $F \subset I$ such that every $V_{j}$ depends on $\prod_{i \in F} S_{i}^{1}$. Let $v=p_{F} \circ u$. Then $\operatorname{Im} v$ $\subset \cup_{j=1}^{m} V_{j}$ and $p_{i_{0}} \circ f \circ u \sim_{h} p_{i_{0}} \circ f \circ v$, hence $h\left(u+B_{1}\right)=h\left(v+B_{1}\right)$. However, $p_{i} \circ v \in B_{1}$ 
for $i \notin F$ and hence $\left(p_{i_{0}} \circ f\right)_{*}\left(v+B_{1}\right)=h\left(v+B_{1}\right)=0$ by the definition of $h$. This contradicts to $h\left(u+B_{1}\right) \neq 0$.

(3) Let $\boldsymbol{T}=\boldsymbol{S}_{1}^{1} \times \boldsymbol{S}_{2}^{1}$ be the torus with base point $\boldsymbol{e}$. Then any endomorphism of $H_{1}(\boldsymbol{T})\left(=H_{1}^{T}(\boldsymbol{T})\right)$ is spatial, but Theorem 6.2 does not hold for $\boldsymbol{T}$-piled spaces. To see this, take free generators $e_{1}, e_{2}, e_{1}^{i}, e_{2}^{i}(i=1,2)$ so that $H_{1}(\boldsymbol{T}) \cong$ $\left\langle e_{1}, e_{2}\right\rangle$ and $H_{1}(\boldsymbol{T} \vee \boldsymbol{T}) \cong\left\langle e_{1}^{1}, e_{2}^{1}\right\rangle \bigoplus\left\langle e_{1}^{2}, e_{2}^{2}\right\rangle$ naturally. Let $h: H_{1}(\boldsymbol{T}) \rightarrow H_{1}(\boldsymbol{T} \vee \boldsymbol{T})$ be the homomorphism such that $h\left(e_{i}\right)=e_{i}^{i}$ for $i=1,2$. Suppose that $h=f_{*}\left(=f_{*}^{T}\right)$ for some continuous map $f: \boldsymbol{T} \rightarrow \boldsymbol{T} \vee \boldsymbol{T}$. Let $p: \boldsymbol{T} \vee \boldsymbol{T} \rightarrow \boldsymbol{S}^{1} \vee \boldsymbol{S}^{1}$ be the projection so that $p$ projects the first torus to the first coordinate $S^{1}$ and the second to the second. Then $p \circ f$ induces a homomorphism from $\pi_{1}(\boldsymbol{T})$ to $\pi_{1}\left(\boldsymbol{S}^{1} \vee \boldsymbol{S}^{1}\right)$ $\left(\cong \boldsymbol{Z} * \boldsymbol{Z}\right.$ ) and $\operatorname{Im} p_{*} h \cong \boldsymbol{Z} \oplus \boldsymbol{Z}$.

(Diagram)

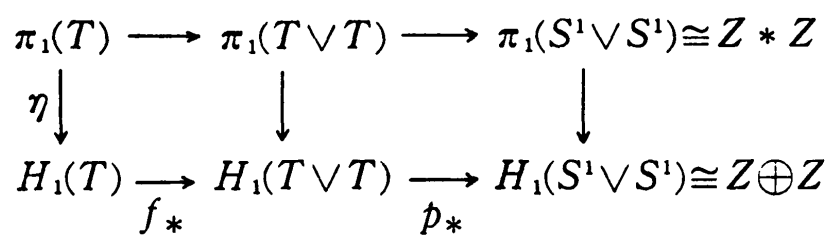

Since any nonzero abelian subgroup of $Z * Z$ is isomorphic to $Z$ and the diagram commutes, $\operatorname{Im} p_{*^{\circ}} f_{*^{\circ} \eta}$ is isomorphic to $Z$ or trivial, hence so is $\operatorname{Im} p_{*} f_{*}$ because $\eta$ is surjective. This is a contradiction.

(4) Let $X$ be a connected 2-simplicial complex and $Y$ a path connected space. Then the standard method shows that any homomorphism $\pi_{1}(X)$ to $\pi_{1}(Y)$ is induced by a continuous map from $X$ to $Y$. On the other hand, there exists a 2 -simplicial complex $X$ with $\pi_{1}(X) \cong Z^{N}$. Then $H_{1}^{T}(X) \cong H_{1}(X) \cong Z^{N}$. However, we cannot replace $\boldsymbol{H}$ by such an $X$ in Thoerem 6.3. In addition, though $H_{1}^{T}\left(\left(\boldsymbol{S}^{1}\right)^{N}\right) \cong Z^{N}$, we cannot replace $\boldsymbol{H}$ by $\left(\boldsymbol{S}^{1}\right)^{\boldsymbol{N}}$ as the preceding (3) shows.

\section{A. Appendix}

All groups in the sequel are abelian groups. Here we prove a hierarchy theorem for the $\sigma$-Reid class of certain abelian groups, which is a version of Theorem 5 of [5] and Theorem 1 of [6] and corresponds to the class of $X$ piled spaces.

Definition A.1. Let $A$ be a group. The $\sigma$-Reid class of $A$ is defined as the smallest class which contains $A$ and also $\tilde{\Pi}_{i \in j} X_{i}$ and $\bigoplus_{i \in I} X_{i}$ when each $X_{i}$ $(i \in I)$ belongs to the class. A group in the $\sigma$-Reid class of $A$ is called a $\sigma$ $\operatorname{Reid}(A)$ group. Along the inductive definition, we define types for $\sigma-\operatorname{Reid}(A)$ groups. Orders are the ordinals of types as in Definition 4.8. 
(1) $\operatorname{ty}(A)=(0, \boldsymbol{P})=(0, \boldsymbol{S})$;

(2) Let $X_{i}(i \in I)$ be $\sigma-\operatorname{Reid}(A)$ groups of type ty $\left(X_{i}\right)$, where $|I| \geqq 2$.

Case (a): $\sup ^{+}\left\{0\left(X_{i}\right): i \in I\right\}=1$.

$$
\begin{aligned}
& \operatorname{ty}\left(\oplus_{i \in I} X_{i}\right)= \begin{cases}(1, \boldsymbol{S}) & \text { if } I \text { is infinite } \\
(0, \boldsymbol{M}) & \text { otherwise. }\end{cases} \\
& \operatorname{ty}\left(\tilde{\Pi}_{i \in I} X_{i}\right)= \begin{cases}(1, \boldsymbol{P}) & \text { if } I \text { is infinite; } \\
(0, \boldsymbol{M}) & \text { otherwise. }\end{cases}
\end{aligned}
$$

Case (b): $\sup ^{+}\left\{o\left(X_{i}\right): i \in I\right\}=\mu+1$ for $\mu \geqq 1$.

$$
\begin{aligned}
& \operatorname{ty}\left(\oplus_{i \in I} X_{i}\right)=\left\{\begin{array}{lr}
(\mu+1, \boldsymbol{S}) & \text { if } I_{\boldsymbol{P}} \text { is infinite; } \\
(\mu, \boldsymbol{S}) & \text { if } I_{\boldsymbol{P}} \text { is empty; } \\
(\mu, \boldsymbol{M}) & \text { otherwise, }
\end{array}\right. \\
& \operatorname{ty}\left(\tilde{\Pi}_{i \in I} X_{i}\right)= \begin{cases}(\mu+1, \boldsymbol{P}) & \text { if } I_{S} \text { is infinite; } \\
(\mu, \boldsymbol{P}) & \text { if } I_{S} \text { is empty; } \\
(\mu, \boldsymbol{M}) & \text { otherwise, }\end{cases}
\end{aligned}
$$

where $I_{P}=\left\{i: \operatorname{ty}\left(X_{i}\right)=(\mu, \boldsymbol{P})\right.$ or $\left.(\mu, \boldsymbol{M})\right\}$ and $I_{S}=\left\{i: \operatorname{ty}\left(X_{i}\right)=(\mu, \boldsymbol{S})\right.$ or $\left.(\mu, \boldsymbol{M})\right\}$. Case (c): $\sup ^{+}\left\{\mathrm{o}\left(X_{i}\right): i \in I\right\}=\mu$ a limit ordinal.

$$
\operatorname{ty}\left(\oplus_{i \in I} X_{i}\right)=(\mu, S) \text { and } \operatorname{ty}\left(\widetilde{\Pi}_{i \in I} X_{i}\right)=(\mu, P) .
$$

(Refer the remarks before Definition 4.8.)

By induction along the definition of $\sigma-\operatorname{Reid}(A)$ groups, we get the following Lemmas.

Lemma A.2. Let $X$ be a $\sigma-\operatorname{Reid}(A)$ group and $(\mu, \Gamma) \leqq \operatorname{ty}(X)$. Then there exists a $\sigma-\operatorname{Reid}(A)$ group of type $(\mu, \Gamma)$ which is isomorphic to a summand of $X$.

LEMmA A.3. If $a \quad \sigma-\operatorname{Reid}(A)$ group $X$ is of type $(\mu, \boldsymbol{M})(\mu \geqq 1)$ then there exist $\sigma-\operatorname{Reid}(A)$ groups $Y_{1}$ and $Y_{2}$ such that $Y_{1} \oplus Y_{2} \cong X$ and $\operatorname{ty}\left(Y_{1}\right)=(\mu, \boldsymbol{P})$ and $\operatorname{ty}\left(Y_{2}\right)=(\mu, S)$.

Since the functors $D$ and $n$ ! not only commute with direct sums but also with $\sigma$-products, we get the next lemma.

Lemma A.4. If $X$ is a $\sigma-\operatorname{Reid}(A)$ group of type $(\mu, \Gamma)$, then there exists a 
$\sigma$-Reid $(A / D(A))$ group of type $(\mu, \Gamma)$ which is isomorphic to $X / D(X)$ and also exists a $\sigma-\operatorname{Reid}(n ! A)$ group of type $(\mu, \Gamma)$ which is isomorphic to $n ! X$.

The next lemma is a version of [14], but there exists no trap of the measurable cardinality, because we only deal with $\sigma$-products. (Direct products cause a trap of the measurable cardinality [11] and [6]. Using $\Pi$ instead of $\tilde{\nabla}$ in in the definition of piled spaces, we get a version of piled spaces. In this case, $H_{n}^{T}$ corresponds to groups in the Reid class, which is obtained by using direct products instead of $\sigma$-products.) We investigate $\sigma$-Reid $(A)$ groups for a group $A$ with the properties $(\boldsymbol{P})$ or $(\boldsymbol{S})$ (Definition 4.9).

LemMA A.5. Let $A_{i}(i \in I)$ be groups and $G_{j}(j \in J)$ be reduced groups. For any homomorphism $h: \tilde{\Pi}_{i \in I} A_{i} \rightarrow \bigoplus_{j \in J} G_{j}(=G)$, there exist finite sets $I^{\prime} \subset I, J^{\prime} \subset J$ and $n \in N$ such that

$$
h\left(n \cdot \tilde{\Pi}_{i \in I \backslash I^{\prime}}, A_{i}\right) \subset \bigoplus_{j \in J^{\prime}} G_{j} .
$$

Proof. Since we want to apply Theorem 1(3) of [8], we use the same notion and notation. There exists a quasi-sheaf $(S, \rho)$ over $\boldsymbol{P}(I)$ such that $S^{\wedge}$ is isomorphic to $\tilde{\Pi}_{i \in I} A_{i}$. (See [8, Definition 1].) By Theorem 1(3) of [8], there exist countably complete maximal filters $F_{1}, \cdots, F_{m}$ of $\boldsymbol{P}(I)$ and an integer $n>0$ and a finite subset $J^{\prime}$ of $J$ such that $h\left(n \cdot K_{F_{1} \cdots F_{m}}\right) \subset \bigoplus_{j \in J^{\prime}} G_{j}$. If $F$ is a non-principal countably complete maximal filter of $\boldsymbol{P}(I) K_{F}=\tilde{\Pi}_{i \in I} A_{i}$ holds. Therefore we may assume that $F_{1}, \cdots, F_{m}$ are principal. There exist $\alpha_{1}, \cdots, \alpha_{m}$ $\in I$ such that $K_{F_{k}}=\tilde{\Pi}_{i \neq \alpha_{k}} A_{i}$. Let $I^{\prime}=\left\{\alpha_{1}, \cdots, \alpha_{m}\right\}$. Then $K_{F_{1} \cdots F_{k}}=\bigcap_{k=1}^{m} K_{F_{k}}=$ $\tilde{\Pi}_{i \in I \backslash I^{\prime}} A_{i}$ and we get the conclusion.

By using Lemma A.5 instead of Lemma 4 of [5], we prove the following lemma.

LEMMA A.6. Let $A$ be a reduced group. If any $\sigma-\operatorname{Reid}(n ! A)$ group of type $(\mu, \Gamma)$ is not isomorphic to a summand of any $\sigma-\operatorname{Reid}(n ! A) \operatorname{group}$ of type $(\mu, \nabla)$ for each $n \in N$, then any $\sigma-\operatorname{Reid}(n ! A)$ group of type $(\mu+1, \nabla)$ is not isomorphic to a summand of any $\sigma-\operatorname{Reid}(n ! A)$ group of type $(\mu+1, \Gamma)$ for each $n \in N$, where $\Gamma=\boldsymbol{P}$ and $\boldsymbol{\nabla}=\boldsymbol{S}$, or $\Gamma=\boldsymbol{S}$ and $\boldsymbol{\nabla}=\boldsymbol{P}$ respectively.

Proof. Suppose that a $\sigma-\operatorname{Reid}(m ! A)$ group $X$ of type $(\mu+1, \nabla)$ is isomorphic to a summand of a $\sigma-\operatorname{Reid}(m ! A)$ group $Y$ of type $(\mu+1, \Gamma)$.

First consider the case that $\Gamma=P$ and $\boldsymbol{V}=\boldsymbol{S}$. Then by Lemma A.3, we may assume that $X=\bigoplus_{i \in I} X_{i}$ and $Y=\tilde{\Pi}_{j \in J} Y_{j}$ where $\left\{i: \operatorname{ty}\left(X_{i}\right)=(\mu, \boldsymbol{P})\right\}$ is infinite and $\operatorname{ty}\left(Y_{j}\right) \leqq(\mu, S)$ for every $j \in J$. There exist $h: X \rightarrow Y$ and $\sigma: Y \rightarrow X$ such 
that $\sigma \circ h=$ id. By Lemma A.5, there exist $n \in N(n>m)$ and finite sets $I^{\prime} \subset I$, $J^{\prime} \subset J$ such that $\sigma\left(n \cdots(m+1) \cdot \tilde{\Pi}_{j \in J \backslash J^{\prime}} Y_{j}\right) \subset \bigoplus_{i \in I^{\prime}} X_{i}$. Then $\bigoplus_{i \in I \backslash I^{\prime}} n \cdots(m+1) X_{i}$ is isomorphic to a summand of $\Pi_{j \in J}, n \cdots(m+1) Y_{j}$. The latter group is isomorphic to a $\sigma-\operatorname{Reid}(n ! A)$ group of type less than or equal to $(\mu, \boldsymbol{S})$. There exists an $i \in I \backslash I^{\prime}$ such that $\operatorname{ty}\left(X_{i}\right)=(\mu, \boldsymbol{P})$. Then $n \cdots(m+1) X_{i}$ is isomorphic to a $\sigma$-Reid $(n ! A)$ group of type $(\mu, \boldsymbol{P})$ and thus we get a contradiction.

Next consider the case that $\Gamma=\boldsymbol{S}$ and $\boldsymbol{V}=\boldsymbol{P}$. Then as the former case, we may let $X=\tilde{\Pi}_{i \in I} X_{i}$ and $Y=\oplus_{j \in J} Y_{j}$ where $\left\{i: \operatorname{ty}\left(X_{i}\right)=(\mu, S)\right\}$ is infinite and $\operatorname{ty}\left(Y_{j}\right) \leqq(\mu, \boldsymbol{P})$ for every $j \in J$. Let $h$ and $\boldsymbol{\sigma}$ be as before. By Lemma A.5, there exist $n \in \boldsymbol{N}(n>m)$ and finite sets $I^{\prime} \subset I, J^{\prime} \subset J$ such that $h(n \cdots(m+1)$. $\left.\tilde{\Pi}_{i \in I \backslash I^{\prime}} X_{i}\right) \subset \bigoplus_{j \in J^{\prime}} Y_{j}$. This implies that $\tilde{\Pi}_{i \in I \backslash I^{\prime}} n \cdots(m+1) X_{i}$ is isomorphic to a summand of $\bigoplus_{j \in J}, n \cdots(m+1) Y_{j}$, which induces a contradiction as the dual case.

Theorem A.7. Let $X$ and $Y$ be $\sigma$-Reid $(A)$ groups and $X$ be isomorphic to a summand of $Y$. Then, $\operatorname{ty}(X) \leqq \operatorname{ty}(Y)$ if $A$ satisfies $(\Gamma)$ and

(a) $\operatorname{ty}(Y)=(n, \nabla)$ for an odd $n \in N$;

(b) $\operatorname{ty}(Y)=(n, \Gamma)$ for an even $n \in \boldsymbol{N}$; or

(c) $o(Y)$ is infinite,

where (1) $\Gamma=\boldsymbol{P}$ and $\boldsymbol{\nabla}=\boldsymbol{S}$; or (2) $\Gamma=\boldsymbol{S}$ and $\boldsymbol{\nabla}=\boldsymbol{P}$, respectively.

Consequently, if $A$ satisfles both $(\boldsymbol{S})$ and $(\boldsymbol{P})$ and $o(Y) \geqq 1$, then $\operatorname{ty}(X) \leqq \operatorname{ty}(Y)$. Therefore, isomorphic $\sigma-\operatorname{Reid}(A)$ groups of nonzero order have the same type.

Proof. If $X$ is isomorphic to a summand of $Y$, then $X / D(X)$ is isomorphic to a summand of $Y / D(Y)$. Therefore we may assume $D(A)=0$ by Lemma A.4.

(Finite case): From Lemma A.6, it suffices to show the case of $n=1$. We use the notation in the proof of Lemma A.6. Suppose that $\operatorname{ty}(X) \equiv \operatorname{ty}(Y)$. If $A$ satisfies $(\boldsymbol{S}), \operatorname{ty}(Y)=(1, \boldsymbol{P})$ and $\operatorname{ty}(X) \geqq(1, \boldsymbol{S})$. By Lemma A.2, we may assume that $X=\oplus_{I} A$ and $Y=\tilde{\Pi}_{J} A$ for infinite index sets $I$ and $J$. By Lemma A.5, there exist $m \in N$ and finite sets $I^{\prime} \subset I, J^{\prime} \subset J$ such that $\sigma\left(m ! \cdot \tilde{\Pi}_{J \backslash J^{\prime}} A\right) \subset \bigoplus_{I^{\prime}} m ! A$. This implies that $\bigoplus_{I \backslash I^{\prime}}(m ! A)$ is isomorphic to a summand of $(m ! A)^{\left|J^{\prime}\right|}$, which contradicts to $(\boldsymbol{S})$. In case $A$ satisfies $(\boldsymbol{P})$, we can perform the proof similarly.

(Infinite case): Suppose that the lemma holds in both cases $\operatorname{ty}(Y)=(\mu, \boldsymbol{P})$ and $\operatorname{ty}(Y)=(\mu, \boldsymbol{S})$. Then it holds in cases $\operatorname{ty}(Y)=(\mu+1, P)$ and $\operatorname{ty}(Y)=(\mu+1, \boldsymbol{S})$ by Lemmas A.6 and A.2. Then it also holds for the case $\operatorname{ty}(Y)=(\mu, \boldsymbol{M})$. The only remaining cases are $\operatorname{ty}(Y)=(\mu, \boldsymbol{P})$ and $\operatorname{ty}(Y)=(\mu, \boldsymbol{S})$ for a limit $\mu$. We 
can prove similarly to Lemma A.6 by using Lemma A.2.

CORRLlary A.8. Let $A$ be a group of finite rank. If $A$ is not isomorphic to a direct sum of a divisible group and a bounded group, then isomorphic $\sigma$ $\operatorname{Reid}(A)$ groups have the same type.

Proof. Since $A / D(A)$ is a group of finite rank and unbounded, $m \cdot A / D(A)$ is a nonzero group of finite rank for each $m \in \boldsymbol{N}$. Therefore $A$ satisfies $(\boldsymbol{P})$ and $(\boldsymbol{S})$. Moreover, $A^{m}$ is isomorphic to $A$ only when $m=1$. Now the corollary follows from Theorem A.7.

There exist many groups of infinite rank which satisfy either $(\boldsymbol{P})$ or $(\boldsymbol{S})$. For example, unbounded almost slender groups satisfy $(\boldsymbol{P})$ and unbounded, reduced Fuchs-44-groups satisfy $(\boldsymbol{S})$. We refer the reader to [7], [10] and [15] fol those groups.

Recall that a group is cotorsion-free if it does not contain a nonzero cotorsion group, and that a homomorphic image of an algebraically compact group is cotorsion [11]. A slender group is cotorsion-free and the class of cotorsionfree groups is closed under direct products and subgroups. To prove a lemma about homomorphisms between $\sigma-\operatorname{Reid}(A)$ groups, we introduce a concept corresponding to basic components of $X$-piled spaces.

DEFINITION A.9. Basic components of $\sigma$ - $\operatorname{Reid}(A)$ groups are inductively defined as follows :

(1) $A$ itself is the only basic component of $A$;

(2) Let $A_{i \lambda}\left(\lambda \in \Lambda_{i}\right)$ be all the basic components of a $\sigma-\operatorname{Reid}(A)$ group $X_{i}$ for each $i \in I$. Then $A_{i \lambda}\left(\lambda \in \Lambda_{i}, i \in I\right)$ are all the basic components of both $\tilde{\Pi}_{i \in I} X_{i}$ and $\bigoplus_{i \in I} X_{i}$.

Note that a basic component of a $\sigma-\operatorname{Reid}(A)$ group $X$ is isomorphic to $A$ and is a subgroup of $X$. The next is a lemma for Theorem 6.2.

Lemma A.10. Let $A$ be a cotorsion-free, and $X$ and $Y$ be $\sigma-\operatorname{Reid}(A) \operatorname{groups}$ and $g, h \in \operatorname{Hom}(X, Y)$. If $g\left|A_{\lambda}=h\right| A_{\lambda}$ for each basic component $A_{\lambda}$ of $X$, then $g=h$.

Proof. We prove by induction on the definition a $\sigma$-Reid $(A)$ group $X$.

(1) In case $X=A$, there is nothing to prove.

(2) In case $X=\bigoplus_{i \in I} X_{i}$, it is clear from $\operatorname{Hom}(X, Y) \cong \Pi_{i \in I} \operatorname{Hom}\left(X_{i}, Y\right)$ and induction hypothesis. In case $X=\tilde{\Pi}_{i \in I} X_{i}$, we can see that $g\left|\bigoplus_{i \in I} X_{i}=h\right| \oplus_{i \in I} X_{i}$ 
by the preceding case. Since $\tilde{\Pi}_{i \in I} X_{i} / \oplus_{i \in I} X_{i}$ is algebraically compact [11, Theorem 42.1] and $Y$ is cotorsion-free, we conclude $g=h$.

In the remaining part of this appendix, we state about $C(X, Z)$ for a scattered subspace $X$ of $\boldsymbol{R}$. First recall definitions concerning scatteredness. For a space $X$, let $X^{\prime}$ be the subset of $X$ consisting of all accumulation points. Let $X_{0}=X$ and define $X_{\alpha}=X_{\beta}^{\prime}$ for $\alpha=\beta+1$ and $X_{\alpha}=\bigcap_{\beta<\alpha} X_{\beta}$ for a limit ordinal $\alpha$. We say that $X$ is scattered if $X_{\alpha}=\varnothing$ for some $\alpha$. For a scattered space $X$, let $r(X)$ be the least ordinal $\alpha$ such that $X_{\alpha}=\varnothing$ and $r(x)=\max \left\{\alpha: x \in X_{\alpha}\right\}$ for $x \in X$.

Proposition A.11. Let $A$ be a group with the discrete topology.

(1) Any $\sigma-\operatorname{Reid}(A)$ group defined by using only index sets of countable cadinalities is isomorphic to $C(X, A)$ for some scattered subspace $X$ of $\boldsymbol{R}$.

(2) For any scattered subspace $X$ of $\boldsymbol{R}, C(X, A)$ is isomorphic to a $\sigma-\operatorname{Reid}(A)$ group defined by using index sets of countable cardinalities.

Proof. (1) To prove by induction, it suffices to realize $\bigoplus_{n \in N} C\left(X_{n}, A\right)$ and $\Pi_{n \in N} C\left(X_{n}, A\right)$ for scattered subspaces $X_{n}$ of $\boldsymbol{R}$. The only nontrivial case is $\oplus_{n \in N} C\left(X_{n}, A\right)$. We may assume $X_{n} \subset(1 / n+1,1 / n)$ and $C\left(X_{n}, A\right) \neq 0$. Let $X=$ $\{0\} \cup \cup_{n \in N} X_{n} \subset \boldsymbol{R}$. Then $X$ is scattered and $C(X, A) \cong A \oplus_{n \in N} C\left(X_{n}, A\right)$. Since every $C\left(X_{n}, A\right)$ contains a summand isomorphic to $A, C(X, A) \cong \bigoplus_{n \in N} C\left(X_{n}, A\right)$.

(2) This is shown by induction on $r(X)$. We remark that for any scattered subspace $X$ of $\boldsymbol{R}, r(X)$ is countable and hence $X$ is countable and 0 -dimensional. If $r(X)=1, X$ is discrete and hence $C(X, A)$ is isomorphic to $A^{X}$, which is a $\sigma-\operatorname{Reid}(A)$ groups. In case $r(X)=\beta+1, X_{\beta}$ is discrete. We can take clopen subsets $U_{x}$ of $X\left(x \in X_{\beta}\right)$ so that $\{x\}=U_{x} \cap X_{\beta}, U_{x} \cap U_{y}=\varnothing$ and $\cup_{x \in X_{\beta}} U_{x}=X$. Now we work in $U_{x}$. Take clopen subsets $V_{n}(n \in N)$ of $U_{x}$ so that $V_{m} \cap V_{n}$ $=\varnothing(m \neq n), \cup_{n \in N} V_{n} \cup\{x\}=U_{x}$ and $\cup_{n \geq n} V_{n} \cup\{x\}(m \in N)$ form neighborhood bases of $x$. Then $C\left(U_{x}, A\right) \cong A \bigoplus \bigoplus_{\pi \in N} C\left(V_{n}, A\right)$, which is isomorphic to a $\sigma$ $\operatorname{Reid}(A)$ group because $r\left(V_{n}\right) \leqq \beta$. Therefore $C(X, A) \cong \Pi_{x \in X} C\left(U_{x}, A\right)$ is also isomorphic to a $\sigma-\operatorname{Reild}(A)$ group. In case $r(X)$ is limit, let $X=\left\{x_{n}: n \in N\right\}$. Since $r\left(x_{n}\right)<r(X)$, we can successively take clopen subsets $U_{n}$ of $X$ so that $U_{i} \cap U_{j}=\varnothing(i \neq j), r\left(U_{n}\right)<r(X)$ and $x_{n} \in \cup_{i=1}^{n} U_{i}$. Then $\cup_{n \in N} U_{n}=X$ and hence $C(X, A) \cong \prod_{n \in N} C\left(U_{n}, A\right)$, which is isomorphic to a $\sigma-\operatorname{Reid}(A)$ group by the induction hypothesis. 


\section{References}

[1] R.A. Alo and H.L. Shapiro, Normal Topological Spaces, Cambridge Univ. Press, London, 1974.

[2] M. G. Barratt and J. Milnor, An example of anomalous singular homology, Proc. Amer. Math. Soc. 13 (1962), 293-297.

[3] A. Dold, Lectures on Algebraic Topology, Springer-Verlag, Berlin-New York, 1972.

[4] M. Dugas and B. Zimmermann-Huisgen, Iterated direct sums and products of modules, LNM 874, pp. 179-193, Springer-Verlage, Berlin-New York, 1981.

[5] K. Eda, On $\boldsymbol{Z}$-kernel groups, Arch. Math. 41 (1983), 289-293.

[6] K. Eda, $\boldsymbol{Z}$-kernel groups of measurable cardinalities, Tsukuba J. Math. 8 (1984), 95-100.

[7] K. Eda, Almost-slender groups and Fuchs-44-groups, Comment. Math. Univ. St. Pauli, 32 (1983), 131-135.

[8] K. Eda, A generalized direct product of abelian groups, J. Algebra, 92 (1985), 33-43.

[9] K. Eda and H. Ohta, On abelian groups of integer-valued continuous functions, their $\boldsymbol{Z}$-duals and $\boldsymbol{Z}$-reflexivity, in Abelian Group Theory, pp. 241-258, Gordon and Greach, New York-London, 1987.

[10] R. Göbel, S. V. Rychov and B. Wald, A general theory of slender groups and Fuchus-44-groups, LMN 874, pp. 194-200, Springer-Verlage, Berlin-New York, 1981.

[11] L. Fuchs, Infinite Abelian Groups, Vol. I, II, Academic Press, New York, 1970, 1973.

[12] M. I. Graev, Free topological groups, Izv. Acad. Nauk SSSR Ser. Mat. 12 (1948), 279-324 (Russian). English transl. Amer. Math. Soc. transl. (1) 8 (1962), 305-364.

[13] S. T. Hu, Theory of Retracts, Wayne State Univ. Press, Detroit, 1965.

[14] A.V. Ivanov, Direct and complete sums of abelian groups, Bull. Moskow Univ., Ser. 1, Math., Vol. 6 (1979), 96 (Russian).

[15] A. V. Ivanov, A problem on abelian groups, Math. USSR Sbornik, 34 (1978), 461474.

[16] S. Kakutani, Uber bie Metrisation der topologischen Gruppen, Proc. Imp. Acad. Tokyo 19 (1936), 82-84.

[17] S. Kakutani, Free topological groups and infinite direct product topological groups, Proc. Imp. Acad. Japan 20 (1944), 595-598.

[18] A. A. Markov, On free topological groups, Izv. Acad. Nauk SSSR Ser. Mat. 9 (1945), 3-64 (Russian). English transl. Amer. Math. Ser. transl. (1) 8 (1962), 195-304.

[19] E. Michael, Uniform AR's and ANR's, Compositio Math. 39 (1979), 129-139.

[20] S.A. Morris, Free abelian topological groups, in Categorical Topology, pp. 375391, ed. by H. L. Bentley et al., Heldermann-Verlag, Berlin, 1984.

[21] P. Nyikos, The theory of nonmetrizable manifolds, in Set Theoretic Topology, ed. by K. Kunen et al., North-Holland, Amstercam, 1984.

[22] W. J. Ralph, An extension of singular homology to Banach algebras, Pacifiic J. Math. 123 (1986), 391-405.

[23] E. H. Spanier, Algebraic Topology, McGraw-Hill, New York-San Francisco, 1966.

[24] M. G. Tkachenko, On completeness of free abelian topological groups, Soviet Math. 
Dokl. 27 (1983), 341-345.

[25] K. Eda, Free $\sigma$-products and noncommutatively slender groups, J. Algebra, to appear.

[26] K. Eda, First countability and local simple connectivity, Proc. Amer. Math. Soc. 109 (1990), 237-241, 